\title{
Ausgabenseitige Effekte der demografischen Entwicklung auf die Kommunalfinanzen
}

\author{
Tim Starke
}

Angenommen: 29. Juni 2021 / Online publiziert: 23. August 2021

(C) Der/die Autor(en) 2021

Zusammenfassung Der demografische Wandel stellt die Kommunen vor zahlreiche Herausforderungen: Darunter sind insbesondere die Effekte der Altersstruktur (ausgabensteigernde Effekte durch Jugendlastigkeit, tendenziell entlastende Effekte durch eine ansteigende Quote an über 65-Jährigen), aber auch sog. Kostenremanenzeffekte zu nennen. Zur empirischen Überprüfung, inwiefern sich die Bevölkerungsstruktur und der Bevölkerungsrückgang auf die kommunalen Haushalte auswirken, wird auf einen Datensatz der kreisangehörigen Gemeinden und kreisfreien Städte Mecklenburg-Vorpommerns im Zeitraum 2000-2016 zurückgegriffen.

Im Ergebnis zeigt sich, dass sich der andauernde Rückgang der Einwohnerzahlen und die verändernde Altersstruktur in den Kommunen Mecklenburg-Vorpommerns zu einem höheren Niveau von Pro-Kopf-Zuschussbeträgen und -Ausgaben führen. So wird zum einen deutlich, dass höhere Anteile von unter 6-Jährigen sowie 6- bis 18-Jährigen zu Mehrbelastungen in den Gemeinden und Städten führen. Die bislang vermehrt in der finanzwissenschaftlichen Literatur beschriebenen Kostenremanenzen werden zum anderen statistisch signifikant nachgewiesen, wobei die Ergebnisse der geografisch gewichteten Regression einen stärkeren Kostenremanenzeffekt in Gemeinden mit einem höheren Bevölkerungsrückgang anzeigen.

Mit Blick auf den Kommunalen Finanzausgleich bietet es sich daher an, die demografischen Sonderbedarfe der Gemeinden gezielt bei der Bestimmung des Finanzbedarfs zu veredeln. Der Bevölkerungsrückgang könnte dabei nach geografischer Lage und somit nach Stärke des Kostenremanenzeffektes unterschiedlich stark berücksichtigt werden.

Dr. Tim Starke $(\bowtie)$

Faculty of Economics and Management Science, University of Leipzig,

Augustusplatz 10, 04109 Leipzig, Deutschland

E-Mail: starke@wifa.uni-leipzig.de 
Schlüsselwörter Demografischer Wandel · Kommunale Bedarfe ·

Kommunalfinanzen · Kommunaler Finanzausgleich · Regressionsanalyse ·

Geografisch gewichtete Regression

\section{Expenditure-side effects of demographic developments on municipal finances}

Abstract Demographic change poses numerous challenges for municipalities.

These include, in particular, the effects of the age structure (expenditure-increasing effects due to a youth bias, tendency-relieving effects due to an increasing proportion of over-65s), but also so-called cost remanence effects. In order to empirically examine the extent to which the population structure and the population decline affect municipal budgets, a data set of the municipalities and independent cities in Mecklenburg-Western Pomerania in the period 2000-2016 is used.

The results show that the ongoing decline in the number of inhabitants and the changing age structure in the municipalities of Mecklenburg-Western Pomerania lead to a higher level of per capita grant amounts and expenditures. On the one hand, it is clear that higher proportions of under-6s and 6- to 18-year-olds lead to additional burdens in the municipalities and cities. On the other hand, the cost remanences that have been increasingly described in the fiscal literature are shown to be statistically significant, with the results of the geographically weighted regression indicating a stronger cost remanence effect in municipalities with a higher population decline.

With regard to the municipal fiscal equalization system, it therefore makes sense to specifically refine the special demographic needs of the municipalities when determining the financial requirements. In this context, population decline could be considered to different extents according to geographic location and thus according to the strength of the cost remanence effect.

Keywords Demographic change $\cdot$ Municipal needs $\cdot$ Municipal finances · Municipal financial equalization · Regression analysis · Geographically weighted regression

\section{Einleitung}

Der demografische Wandel stellt die kommunalen Haushalte vor vielgestaltige Herausforderungen. Dabei spielen verschiedene Faktoren eine Rolle, darunter vor allem Kostenremanenzeffekte sowie die Kinder- und Jugendlastigkeit der kommunalen Ausgaben. Gleichwohl sind auch die kommunalen Einnahmen umfassend von der demografischen Entwicklung betroffen: Insbesondere der sinkende Anteil der potenziell erwerbstätigen Bevölkerung kann hierbei zu sinkenden Steuereinnahmen führen, welche die kommunale Einnahmenbasis insgesamt schmälern. Aus diesem Grund stehen den Kommunen weniger Einnahmen zur Verfügung, um die aus der Wahrnehmung ihrer vielschichtigen Aufgaben hervorgehenden Ausgaben zu decken. Dementsprechend nehmen die Zuweisungen der Länder (und des Bundes) eine es- 
senzielle Rolle ein, um den kommunalen Haushalten eine finanzielle Mindestausstattung zu garantieren.

In diesem Zusammenhang findet seit Jahrzehnten eine Diskussion innerhalb der finanzwissenschaftlichen Literatur statt, ob es möglich ist, die kommunalen Finanzund Ausgabenbedarfe objektiv zu bestimmen (ausführlicher Gottl-Ottlilienfeld 1928; Ewringmann 1971; Kops 1989; Junkernheinrich 1992; Hesse und Starke 2018; Starke 2020). Auch die Folgen des demografischen Wandels gehen in diese Diskussion mit ein: Hierbei steht vor allem die Frage im Mittelpunkt, ob Gemeinden mit einer absinkenden Einwohnerzahl statistisch signifikante finanzielle Mehrbelastungen verzeichnen und wie die dadurch entstehenden kommunalen Mehrbedarfe finanziell aufgefangen werden können. Der Einfluss der demografischen Entwicklung auf die kommunalen Haushalte soll anhand eines Datensatzes der Gemeinden und Städte Mecklenburg-Vorpommerns 2000-2016 empirisch überprüft werden, wobei einerseits der Rückgang der Bevölkerung und andererseits die Veränderung der Altersstruktur im Fokus des Beitrags stehen. Bezüglich der Frage nach dem finanziellen Ausgleich etwaiger Mehrbelastungen wird im Anschluss daran auf die essenzielle Rolle des Kommunalen Finanzausgleichs eingegangen, der den Kommunen eine aufgabenangemessene Finanzausstattung zur Verfügung stellen muss.

$\mathrm{Zu}$ Beginn sollen in Kap. 2 zunächst der Rückgang der Bevölkerung und die Veränderung der Altersstruktur in den Kommunen nachvollzogen werden, um die Bedeutung des demografischen Wandels zu untermauern. Im darauffolgenden Kap. 3 werden die ausgabenseitigen Effekte des demografischen Wandels näher beleuchtet. Im Fokus stehen dabei die Herleitung und Klassifizierung von Kostenremanenzeffekten sowie die Erstellung von Altersstrukturkostenprofilen für die gemeindliche Ebene Mecklenburg-Vorpommerns. In Kap. 4 werden die empirischen Untersuchungen durchgeführt: Dazu wird zunächst ein Fixed Effects-Regressionsmodell aufgestellt und dessen Ergebnisse ausführlich interpretiert. Zudem wird eine geografisch gewichtete Regression durchgeführt, die aufzeigen soll, ob die Kostenremanenzeffekte von der topografischen Lage einer Gemeinde abhängig sind. Anschließend wird in Kap. 5 aufgegriffen, wie der demografische Wandel bisher in den Kommunalen Finanzausgleichssystemen anerkannt wird. Auf Basis der Ergebnisse der empirischen Analysen wird zudem eruiert, ob es alternative Wege gibt, die schrumpfenden Einwohnerzahlen bei der Bestimmung des Finanzbedarfs im Schlüsselzuweisungssystemen zu berücksichtigen. Die wichtigsten Erkenntnisse werden abschließend in einem Fazit zusammengefasst und ein Ausblick gegeben.

\section{Demografische Entwicklung Mecklenburg-Vorpommerns}

Die Kommunen in Mecklenburg-Vorpommern sind im bundesweiten und auch im ostdeutschen Vergleich stark vom demografischen Wandel betroffen. Wichtig ist dabei, dass sich die Entwicklungen sowohl in einem Rückgang der Einwohnerzahl (Abschn. 2.1) als auch in einer Veränderung der Altersstruktur (Abschn. 2.2) auswirken. Beide Faktoren sollen nachfolgend betrachtet und in das Verhältnis zur bundesweiten Entwicklung gesetzt werden, um die Besonderheiten MecklenburgVorpommerns hervorzuheben. Daran anknüpfend wird in Vorbereitung auf die geo- 
grafisch gewichtete Regression ein interkommunaler Vergleich vorgenommen, um unterschiedliche Entwicklungstrends zwischen Landkreisen, kreisfreien Städten und großen kreisangehörigen Gemeinden herauszuarbeiten.

\subsection{Bevölkerungsrückgang}

Tab. 1 gibt einen Überblick über die Entwicklung der Einwohnerzahlen verschiedener Vergleichsgruppen über den Zeitraum von 2000 bis 2016. Dabei fällt auf, dass die Einwohnerzahl Mecklenburg-Vorpommerns um 9,3\% abgenommen hat, was etwa der durchschnittlichen Entwicklung der Einwohnerzahl in den ostdeutschen

Tab. 1 Entwicklung der Bevölkerung in Deutschland und Mecklenburg-Vorpommern 2000-2016 im Vergleich

\begin{tabular}{|c|c|c|c|c|c|c|c|}
\hline [in Tsd. Einwohnern] & 2000 & 2004 & 2008 & 2012 & 2016 & $\begin{array}{l}V R \\
2016 / 2000 \\
(\%)\end{array}$ & $\begin{array}{l}V R \\
2016 / 2012 \\
(\%)\end{array}$ \\
\hline $\begin{array}{l}\text { Bundesrepublik } \\
\text { Deutschland }\end{array}$ & 82.260 & 82.501 & 82.002 & 80.524 & 82.522 & $+0,3$ & $+2,5$ \\
\hline Flächenländer West & 62.652 & 63.282 & 63.107 & 62.230 & 63.876 & $+2,0$ & $+2,6$ \\
\hline Flächenländer Ost & 13.850 & 13.433 & 13.029 & 12.530 & 12.581 & $-9,2$ & $+0,4$ \\
\hline Mecklenburg-Vorpommern & 1776 & 1720 & 1664 & 1600 & 1611 & $-9,3$ & $+0,6$ \\
\hline Kreisfreie Städte & 302 & 296 & 297 & 294 & 303 & $+0,5$ & $+3,1$ \\
\hline $\begin{array}{l}\text { Große kreisangehörige } \\
\text { Städte }\end{array}$ & 235 & 225 & 223 & 219 & 224 & $-4,8$ & $+2,2$ \\
\hline $\begin{array}{l}\text { Kreisangehörige } \\
\text { Gemeinden }\end{array}$ & 1239 & 1198 & 1145 & 1087 & 1084 & $-12,5$ & $-0,3$ \\
\hline Rostock, Hansestadt & 201 & 199 & 201 & 203 & 208 & $+3,5$ & $+2,3$ \\
\hline $\begin{array}{l}\text { Schwerin, } \\
\text { Landeshauptstadt }\end{array}$ & 101 & 97 & 96 & 91 & 96 & $-5,5$ & $+4,8$ \\
\hline Neubrandenburg, Stadt & 73 & 68 & 66 & 64 & 64 & $-13,0$ & $+0,4$ \\
\hline Stralsund, Hansestadt & 61 & 59 & 58 & 57 & 59 & $-2,6$ & $+3,0$ \\
\hline Wismar, Hansestadt & 47 & 45 & 45 & 42 & 43 & $-8,6$ & $+1,3$ \\
\hline Greifswald, Hansestadt & 54 & 53 & 54 & 56 & 58 & $+6,9$ & $+4,0$ \\
\hline $\begin{array}{l}\text { Landkreis } \\
\text { Mecklenburgische } \\
\text { Seenplatte }\end{array}$ & 309 & 294 & 279 & 264 & 262 & $-15,3$ & $-0,9$ \\
\hline Landkreis Rostock & 231 & 227 & 219 & 211 & 214 & $-7,2$ & $+1,5$ \\
\hline $\begin{array}{l}\text { Landkreis Vorpommern- } \\
\text { Rügen }\end{array}$ & 255 & 245 & 235 & 224 & 225 & $-11,8$ & $+0,6$ \\
\hline $\begin{array}{l}\text { Landkreis Nordwest-Meck- } \\
\text { lenburg }\end{array}$ & 168 & 166 & 163 & 156 & 157 & $-6,8$ & $+0,7$ \\
\hline $\begin{array}{l}\text { Landkreis Vorpommern- } \\
\text { Greifswald }\end{array}$ & 270 & 258 & 250 & 239 & 237 & $-12,2$ & $-0,8$ \\
\hline $\begin{array}{l}\text { Landkreis Ludwigslust- } \\
\text { Parchim }\end{array}$ & 241 & 233 & 223 & 212 & 213 & $-11,6$ & $+0,1$ \\
\hline
\end{tabular}

* VR= Veränderungsrate

Quelle: Eigene Darstellung, eigene Berechnungen. Daten: Statistisches Bundesamt (GENESIS OnlineDatenbank) und Statistisches Amt Mecklenburg-Vorpommern, Bevölkerungsstand jeweils zum 31.12. 
Flächenländern entspricht. Für den bundesweiten Durchschnitt ergibt sich hingegen ein leichtes Wachstum von 0,3\%. Bezogen auf den kürzeren Zeitraum von 2012 bis 2016 sind die Effekte der Flüchtlingszuwanderung zu beachten, die teilweise für ein erhebliches Bevölkerungswachstum in den Ländern und darunter auch MecklenburgVorpommern verantwortlich sind.

Innerhalb Mecklenburg-Vorpommerns verzeichnen die kreisfreien Städte im Durchschnitt ein leichtes Wachstum von $0,5 \%$, wobei lediglich die Einwohnerzahl von Rostock angestiegen ist (+3,5\% ggü. 2000), während die Landeshauptstadt Schwerin hingegen schrumpfte (-5,5\% ggü. 2000). Die großen kreisangehörigen Städte büßten im Gesamtzeitraum etwa $4,8 \%$ ihrer Einwohnerzahl ein. Während Neubrandenburg, Stralsund und Wismar einen geringfügigen bis starken Bevölkerungsrückgang hinnehmen mussten, stieg die Einwohnerzahl in Greifswald um $6,9 \%$, was vorrangig auf ihren Status als Universitätsstadt zurückgeführt werden kann. Die Entwicklung in den (kleinen) kreisangehörigen Gemeinden verdeutlicht, dass der ländliche Raum Mecklenburg-Vorpommerns einen deutlich höheren Bevölkerungsrückgang verzeichnet $(-12,5 \%)$. Überdies wird deutlich, dass sich die Bevölkerungsentwicklung seit 2012 zudem relativ stabilisiert hat. Hier ist Schwerin hervorzuheben, wo die Einwohnerzahl 2016 um 4,8\% gegenüber 2012 gestiegen ist.

Die Heterogenität des demografischen Wandels verdeutlicht sich mit Blick auf eine Kartendarstellung (Abb. 1): In der Nähe der Oberzentren Rostock, Schwe-

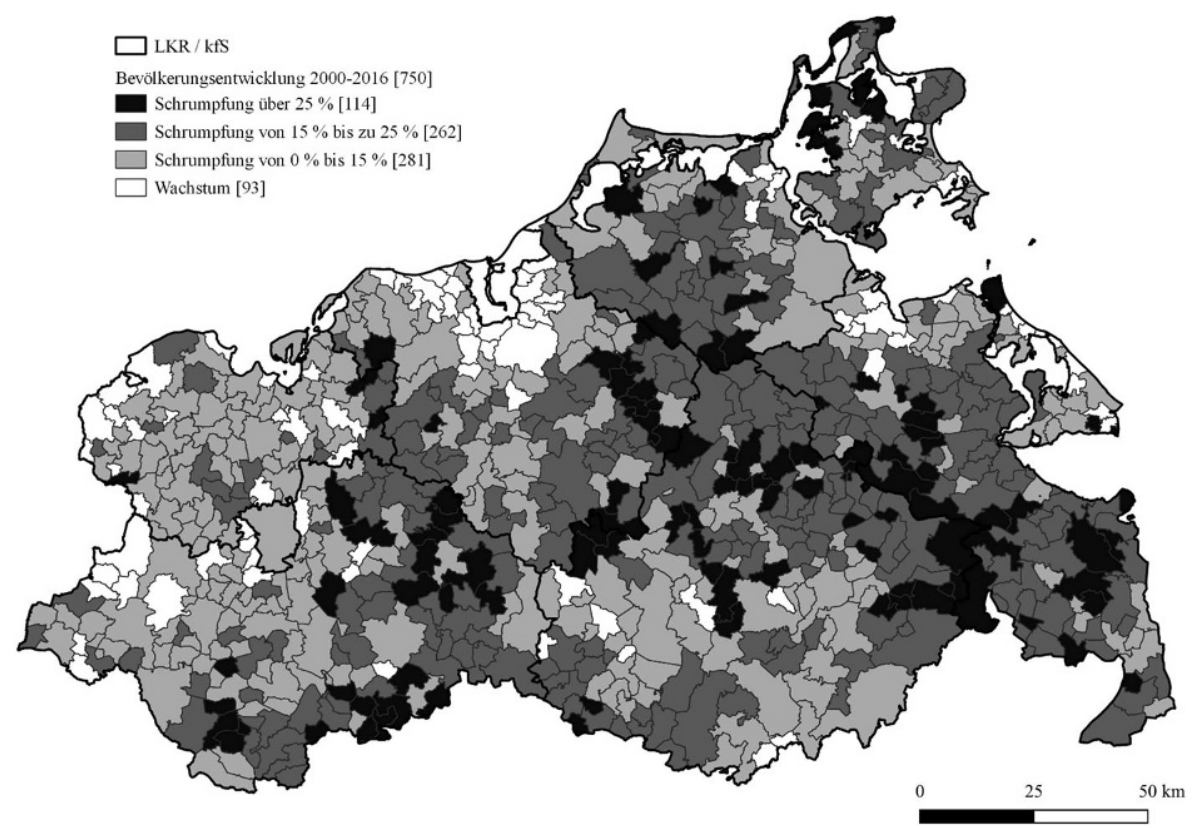

Abb. 1 Veränderungsraten der Einwohnerzahlen der kreisangehörigen Gemeinden und kreisfreien Städte Mecklenburg-Vorpommerns von 2000-2016. Quelle: Eigene Darstellung, eigene Berechnungen. Daten: Statistisches Amt Mecklenburg-Vorpommern, Bevölkerungsstand jeweils zum 31.12., Herausgeber der Kartengrundlage: GeoBasis-DE/MV; dl-de/by-2-0 
rin, Greifswald und Stralsund befindliche Gemeinden können i.d.R. steigende Einwohnerzahlen vorweisen. Lediglich auf Neubrandenburg und seine Umlandgemeinden trifft dies nicht zu. Mittelzentren, wie bspw. Wismar und Güstrow, oder auch Grundzentren, z. B. Torgelow oder Sassnitz, weisen nur geringe Zugewinne an Bevölkerung oder sogar Einwohnerrückgänge auf. Die restlichen kreisangehörigen Gemeinden schrumpfen i.d.R. deutlich. Besonders an der Grenze zwischen den Landkreisen Mecklenburgische Seenplatte und Vorpommern-Greifswald dominieren negative Veränderungsraten der Einwohnerzahlen in den entsprechenden Gemeinden. Dementsprechend ist im Hinblick auf die empirischen Untersuchungen zu erwarten, dass in diesen Gemeinden die stärksten Anpassungen an den demografischen Wandel erforderlich sein werden. Generell sinkt der Bevölkerungsstand im Osten Mecklenburg-Vorpommerns stärker als im Westen. Mithin verzeichnen viele Städte in Grenznähe zum Oberzentrum Lübeck positive Entwicklungstrends.

Des Weiteren zeigt sich, dass benachbarte Gemeinden bzw. Regionen gegensätzlichen Wachstumsdynamiken und somit unterschiedlichen Anforderungen an den demografischen Wandel ausgesetzt sind (Wissenschaftlicher Beirat beim Bundesministerium der Finanzen 2013, S. 11). Dies wirkt sich auf die Siedlungsentwicklung aus, da Gemeinden mit stark sinkenden Einwohnerzahlen i.d.R. eine Unterauslastung ihrer Infrastruktur und Siedlungsfläche vorweisen, während in wachsenden Gemeinden Mehrbedarfe an Siedlungsfläche und Infrastruktur entstehen. Die Kluft zwischen Gemeinden mit unterschiedlichen Dynamiken von Einwohnerzahl und Siedlungsflächen sowie von Ausgaben für den Aufbau und die Unterhaltung von Infrastruktur vergrößert sich folglich und führt zu höheren Ausgaben je Einwohner (ausführlicher Siedentop et al. 2015, S. 4).

\subsection{Altersstruktur}

Für die Finanzierung des öffentlichen Angebots an Gütern und Dienstleistungen ist auch die Entwicklung der Bevölkerungsstruktur relevant. Dazu wird ersichtlich, dass die Verschiebung der Altersstruktur in Mecklenburg-Vorpommern im Zeitraum von 2000-2016 recht homogen verlief (Abb. 2).

So hat der Anteil an unter 6-Jährigen leicht zugenommen (+1,0 Prozentpunkte), der Anteil an 6- bis 18-Jährigen hingegen deutlich abgenommen (-4,5 Prozentpunkte). Der Anteil der erwerbsfähigen Bevölkerung ist ähnlich stark zurückgegangen

Abb. 2 Vergleich der Altersstruktur der Bevölkerung Mecklenburg-Vorpommerns für die Jahre 2000 und 2016. Quelle: Eigene Darstellung, eigene Berechnungen. Daten: Statistisches Amt Mecklenburg-Vorpommern, Bevölkerungsstand jeweils zum 31.12.

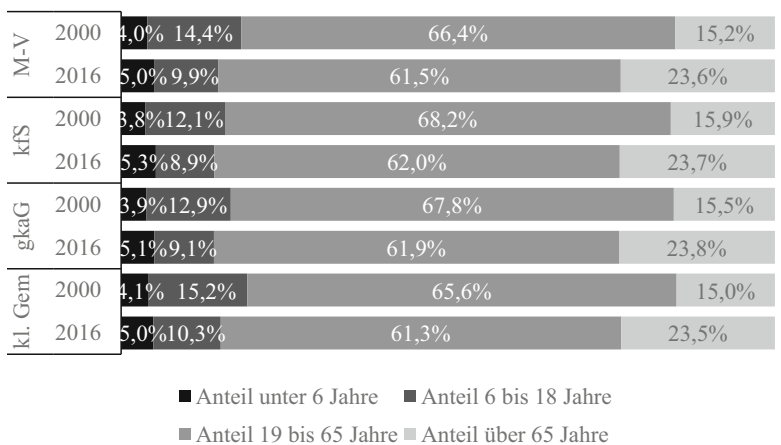


(-4,9 Prozentpunkte), während der Prozentsatz der über 65-Jährigen insgesamt am stärksten zugenommen hat $(+8,4$ Prozentpunkte). Dabei verzeichnen die kleinen kreisangehörigen Gemeinden den geringsten Zuwachs bei der unter 6-jährigen Bevölkerung (+0,8\%), die kreisfreien Städte hingegen den höchsten $(+1,6 \%)$, wodurch diese 2016 mit 5,3\% den höchsten Anteil aufweisen. Der Anteil der 6- bis 18-Jährigen ist hingegen wiederum in den kreisangehörigen Gemeinden mit 10,3\% am höchsten. Wichtig ist hierbei, dass der alleinige Blick auf die Altersstruktur bezüglich der Entwicklung von Ausgaben und Zuschussbeträgen nicht ausreicht. So sind Rostock und Schwerin Träger weiterführender Schulen (Gymnasien oder Berufsschulen), die auch von einpendelnden Schülern besucht werden, welche nicht in die eigene Altersstruktur eingehen. Die daraus entstehenden Mehrausgaben werden wiederum durch Einnahmen aus der Schulumlage vergolten (ausführlicher Lenk et al. 2017, S. 218). Der Rückgang des Anteils der Bevölkerung im Alter von 19 bis 65 Jahren fiel indes in den kreisfreien Städten am höchsten aus (-6,2 Prozentpunkte), der höchste Anstieg des Anteils der über 65-Jährigen kann mit 9,4 Prozentpunkten im kreisangehörigen Raum (ohne die großen kreisangehörigen Gemeinden) beobachtet werden. Dies verdeutlicht, dass die kommunalen Teilräume Mecklenburg-Vorpommerns zwar unterschiedlich stark vom demografischen Wandel betroffen sind, jedoch ähnliche Effekte auf der Ausgabenseite zu erwarten sind: Rein quantitativ betrachtet sind im bedeutsamen Aufgabenbereich der Kindertagesstätten und bei der altersorientierten Aufgabenwahrnehmung belastende Effekte, hinsichtlich der jugendorientierten Ausgaben hingegen entlastende Wirkungen zu erwarten.

\section{Kostenremanenzen und Altersstrukturkosten}

Der demografische Wandel beeinflusst sowohl die Ausgaben- als auch die Einnahmenseite der kommunalen Finanzen. Einnahmenseitig sind hierbei gleichbleibende bzw. steigende Pro-Kopf-Gebühren bei standortgebundenen Infrastrukturen zu nennen (z. B. Abfall- und Abwasserbeseitigung und Wasserversorgung). Hinsichtlich der Steuereinnahmen werden die gewinnabhängigen Steuerarten (z. B. Gewerbesteuer) nur geringfügig vom demografischen Wandel beeinflusst. Beim Gemeindeanteil an der Einkommensteuer wurde jedoch ein Einfluss der Bevölkerungsentwicklung nachgewiesen: So stiegen die Einnahmen in schrumpfenden Gemeinden im Zeitverlauf statistisch signifikant langsamer an als in Gemeinden mit einer wachsenden Bevölkerungszahl (Starke 2020, S. 174ff.). Derartige Einnahmenverluste müssen dann in der Folge mittels Zuweisungen (bspw. aus dem Kommunalen Finanzausgleich) aufgefangen werden (vgl. Abschn. 5). In Bezug auf die ausgabenseitigen Effekte werden generell die sog. Kostenremanenzeffekte und deren Auswirkungen auf die kommunalen Haushalte in der vorherrschenden Literatur zum demografischen Wandel untersucht (Abschn. 3.1). Allerdings ist nicht von einem allgemeingültigen Zusammenhang zwischen der Entwicklung der öffentlichen Gesamtausgaben und der Einwohnerzahl auszugehen. Vielmehr führt auch die Veränderung der Altersstruktur zu Verschiebungen in der Ausgabenstruktur. Die sich daraus ergebenden Belastungspotenziale können mit Hilfe von Altersstrukturkostenprofilen quantifiziert werden (Abschn. 3.2). 


\subsection{Kostenremanenzeffekte}

Mit Bezug auf die meisten kommunalen Aufgabenbereiche steigen die Gesamtausgaben im Falle eines Bevölkerungswachstums nicht proportional an. Stattdessen können unter Umständen Kostendegressionseffekte entstehen, weil sich die fixen Kosten der Leistungserstellung auf eine größere Anzahl an Bürgern verteilen (Lenk und Starke 2015, S. 5). Demgegenüber führt das mit dem demografischen Wandel einhergehende Absinken der Einwohnerzahl zu sog. Kostenremanenzeffekten, weil sich der Rückgang der Einwohnerzahl ,,[...] in den für die Nutzung bzw. Finanzierung relevanten Altersgruppen schneller vollzieht als eine für die Kommune und die Nutzer realisierbare sowie rechtlich zulässige Anpassung der infrastrukturellen und organisatorischen Strukturen der Aufgabenerfüllung vollzogen werden kann [...]“" (ausführlicher Dobroschke et al. 2014, S. 44). Deshalb reduziert sich das aufgabenspezifische Defizit in einem gemeindlichen Haushalt langsamer als die Einwohnerzahl in den entsprechenden Alterskohorten (sog. milde Remanenz). Im mittleren Fall könnte das Defizit (in Absolutgrößen) gleichbleiben und im Extremfall sogar ansteigen (Abb. 3). Mit anderen Worten kann zwar die Nachfrage nach einer altersspezifischen Leistungserstellung in einer schrumpfenden Gemeinde sinken, jedoch können die Gesamtausgaben nicht in gleichem Maße zurückgeführt werden wie die Einwohnerzahl (Lenk und Starke 2015, S. 5).

Kostenremanenzen entstehen aufgrund der hohen, unteilbaren Fixkosten bei der öffentlichen Leistungserstellung (ausführlicher Eck et al. 2012, S. 23; Bogumil et al. 2013, S. 260), worunter vor allem Betriebskosten, Kosten der Mindestpersonalausstattung und Kapitalkosten der Infrastruktur zu verstehen sind (Lenk et al. 2012b, S. 105). Ein Gegensteuern ist hierbei nur begrenzt möglich, da es sich im Regelfall um sprungfixe Kosten handelt, die teilweise unabhängig von der Einwohnerzahl der Gemeinde sind (Sachverständigenrat zur Begutachtung der Gesamtwirtschaftlichen Entwicklung 2004, S. 669; Büttner et al. 2008, S. 158). Demzufolge entspricht die

Abb. 3 Ausprägung und Intensität von Kostenremanenzeffekten. Quelle: Eigene Darstellung nach Seitz et al. 2004, S. 83 und Lenk et al. 2018, S. 61

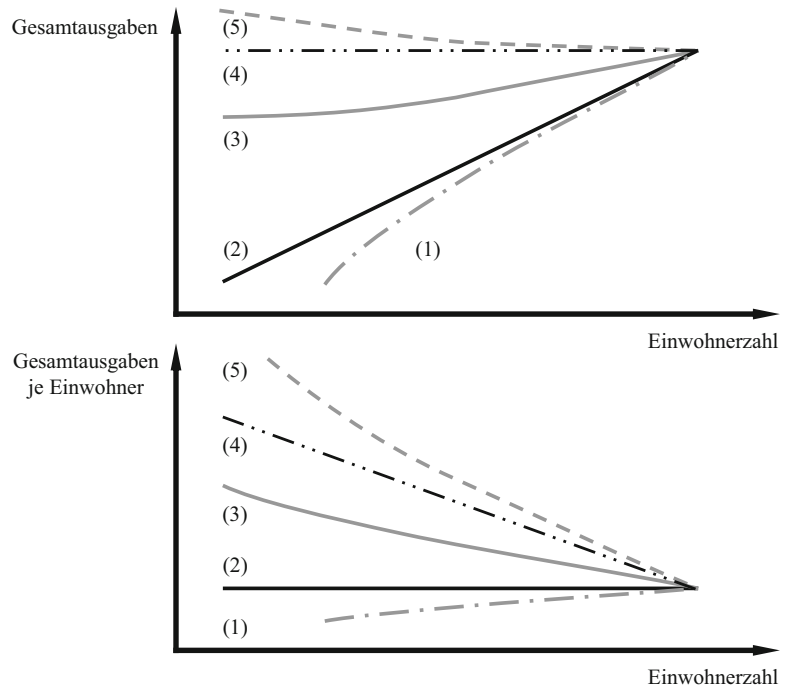


Anpassungsfähigkeit der kommunalen Infrastruktur einem essenziellen Kriterium: Sofern die Kosten pro Nachfrager auch Nachfragerückgang ungefähr konstant gehalten werden können, lassen sich Kostenremanenzeffekte vermeiden (Gutsche 2006, S. 271).

Werden die Entwicklung der Einwohnerzahl und der Ausgaben insgesamt (oben) sowie der Pro-Kopf-Ausgaben (unten) gegenübergestellt, ergeben sich insgesamt fünf Fälle:

- Fall (1) zeigt Kostendegressionseffekte im Falle eines Bevölkerungsrückgangs: Sinkt die Einwohnerzahl um 1\%, dann sinken die Ausgaben um mehr als 1\%. Das Resultat sind folglich rückläufige Pro-Kopf-Ausgaben. Wächst die Einwohnerzahl jedoch um $1 \%$, erhöhen sich die Ausgaben um mehr als $1 \%$, wodurch die Ausgaben je Einwohner ansteigen.

- In Fall (2) treten weder Kostenremanenz- noch Kostendegressionseffekte auf. Dementsprechend könnten die Ausgaben im Falle eines Einwohnerrückgangs proportional und vollständig elastisch absinken (45-Linie). Eine 1-prozentige Verringerung der Einwohnerzahl würde eine 1-prozentige Absenkung der Ausgaben mit sich bringen, wodurch sich die Pro-Kopf-Ausgaben gleichbleibend fortentwickeln. Dies setzt eine unendlich schnelle Anpassungsgeschwindigkeit der lokalen Entscheidungsträger an sich verändernde Rahmenbedingungen aufgrund des demografischen Wandels voraus und gilt daher als unrealistisches Szenario.

- Fall (3) beschreibt milde Kostenremanenzen, in deren Folge die Gesamtausgaben bei rückläufiger Einwohnerzahl nur unterproportional sinken und der Bevölkerungsrückgang im kommunalen Haushalt nicht vollständig kompensiert werden kann. Ein 1-prozentiger Rückgang der Einwohnerzahl führt demnach zu einem Absinken der kommunalen Ausgaben zwischen 0 und 1\%. Basierend darauf liegt die Zunahme der Pro-Kopf-Ausgaben zwischen 0 und 1\%. Die Ausgabenelastizität der Bevölkerungsentwicklung tendiert also in den betroffenen Aufgabenbereichen zwischen 0 und -1 (Freigang 2012, S. 92).

- Fall (4) zeigt gleichbleibende Ausgaben in Absolutgrößen, wodurch diese nicht von der Veränderung der Einwohnerzahl abhängig sind. Das bedeutet jedoch wiederum, dass die Ausgaben je Einwohner im Falle eines Rückgangs bzw. eines Anstiegs der Einwohnerzahl linear zunehmen bzw. abnehmen. Die Ausgabenelastizität der Bevölkerungsentwicklung beläuft sich auf genau -1 .

- Fall (5) skizziert erhebliche Kostenremanenzen, bei denen die Ausgaben der kommunalen Leistungserstellung entweder stagnieren oder sogar ansteigen. Ein 1-prozentiges Absinken der Einwohnerzahl bewirkt einen mehr als 1-prozentigen Anstieg der Gesamtausgaben und demnach massiv steigende Ausgaben je Einwohner. Folglich liegt die Ausgabenelastizität der Bevölkerungsentwicklung (betraglich) über -1 .

Üblicherweise werden technische und politische Kostenremanenzen unterschieden: Erstere gehen bei sinkender Nutzerzahl mit steigenden Ausgaben je Einwohner einher (Gnädinger 2012, S. 28) und betreffen die öffentliche Verwaltung sowie die Schaffung und den Erhalt technischer Infrastruktur im Rahmen der Daseinsvorsorge. Beispielhaft sind hier das Straßennetz, Anlagen des ÖPNV und (speziell leitungs- 
gebundene) Versorgungs- und Entsorgungsleistungen zu nennen (Lenk et al. 2012a, S. 79; Grüttner und Hesse 2013, S. 54). Insbesondere Wasser- und Abwasserleitungen werden langfristig installiert und sind auf eine bestimmte Einwohnerzahl sowie Verbrauchswerte je Einwohner ausgelegt. Verringert sich die Nutzeranzahl oder die Nachfrage nach Trinkwasser, ist der Rückbau der Infrastruktur nicht in gleichem Maße möglich. Neben der Entwicklung der Anzahl bestimmter Nachfrager bzw. Nutzer spezifischer kommunaler Infrastruktur ist auch die Entwicklung der Gesamtbevölkerung entscheidend, da bestimmte kommunale Pflicht- und Weisungsaufgaben in der Fläche erbracht und aufgrund vorgegebener Standards unabhängig von der Nachfrage in einem bestimmten Mindestumfang vorzuhalten sind. Sinkt die Einwohnerzahl, dann steigen über den Transmissionsweg einer abnehmenden Bevölkerungsdichte die Kosten je Einwohner für diese Infrastrukturen. Dabei sind die größten Kostenanstiege in dünn besiedelten Regionen zu vermuten (Lenk et al. 2012a, S. 79f.).

Politische Kostenremanenzeffekte entstehen vor allem in sozialer Infrastruktur, wie Bädern, Museen, Theatern, Bibliotheken und vor allem Schulen (Wissenschaftlicher Beirat beim Bundesministerium der Finanzen 2013, S. 24), wobei speziell letztere bereits eingehend in der Literatur beschrieben wurden: So steigen die ProKopf-Ausgaben für Schulträgeraufgaben im Falle sinkender Schülerzahlen an, weil sich für den Stromverbrauch, die Heizungskosten und die Hausverwaltung keine Einsparpotenziale ergeben (Seitz et al. 2004, S. 83; Hauff und Tarkan 2006, S. 17). Eine Schließung des Gebäudes hätte zwar zur Folge, dass die pro Nachfrager anfallenden Kosten nicht zu stark ansteigen und die Auslastung an Schülern in anderen Schulen innerhalb der Region ansteigt (Gutsche 2006, S. 273). In der Öffentlichkeit werden solche Maßnahmen jedoch als schmerzhafte Eingriffe in die öffentlichen Aufgabenerfüllung wahrgenommen (Bauer und Frei 2017, S. 21), weil dies einen Abbau von Lehrpersonal nach sich zieht und andere schulische Einrichtungen darüber hinaus unzumutbar weit entfernt sein können (Seitz 2004, S. 10; Bogumil et al. 2013, S. 260). Überdies wäre im Vorfeld einer Schulschließung zusätzlich zu prüfen, wie sich die Kosten der Schülerbeförderung entwickeln würden, wenn sich dadurch bspw. weitere Schulwege und eine steigende Anzahl an zu befördernden Schülern ergeben. Zweifellos führen derartige Entscheidungen zu Widerständen bei den Bürgern, was eine Durchsetzung von Anpassungen durch politische Entscheidungsträger enorm erschwert. Deshalb werden schulische Einrichtungen auch bei erheblich sinkenden Schülerzahlen mit steigenden Ausgaben je Schüler erhalten, da eine Schließung politisch nicht durchsetzbar erscheint.

Kostenremanenzeffekte treten schleichend und unauffällig auf: Erst mit einer zeitlichen Verzögerung wird den Entscheidungsträgern die Problematik bewusst, weil der Bevölkerungsrückgang einen langwierigen Prozess darstellt und die Pro-KopfAusgaben nicht sprunghaft, sondern langsam und stetig wachsen. Hinzu kommt das Problem, dass die Haushaltspläne der Kommunen nur in Absolut- und nicht in Pro-Kopf-Größen aufgestellt werden, wobei die Planansätze von Ausgabepositionen i.d.R. vereinfachend über die Zeit hinweg fortgeschrieben werden. Diese Vorgehensweise begünstigt die Intransparenz von Kostenremanenzen für die Politik nochmals (Seitz 2004, S. 10; Lenk und Starke 2015, S. 6). Zudem ist der Abbau von Kostenremanenzen wegen bereits umgesetzter Planungs- und Investitionsent- 
scheidungen für eine Gemeinde i.d.R. kaum möglich ist (Bundesministerium für Verkehr, Bau und Stadtentwicklung (BMVBS) 2007, S. 77).

Nationale empirische Studien zeigen ein recht eindeutiges Bild hinsichtlich des Auftretens von Kostenremanenzeffekten: So stellten Frei et al. (2018) mittels einer OLS-Querschnitt-Längsschnitt-Analyse fest, dass in Städten mit mehr als 20.000 Einwohnern im Zeitraum von 1950-2012 Kostenremanenzen in den Gesamtausgaben sowie nach Aufgabenbereichen auftreten. Ein 1-prozentiger Rückgang der Einwohner würde demnach ceteris paribus (c.p.) zu einem Anstieg der Gesamtausgaben je Einwohner um 1,674\% führen. Ähnlich argumentieren Seitz et al. (2004), die Kostenremanenzen in den ostdeutschen Kreisen 1995-2001 nachwiesen. Auch das BMVBS (2007) konnte Kostenremanenzeffekte am Beispiel der Kommunen Brandenburgs im Zeitraum von 1997-2004 nachweisen, u.a. in den Aufgabenbereichen der öffentlichen Verwaltung, den Schulen sowie dem Bauund Wohnungswesen. Siedentop et al. (2006) zeigten, dass Kostenremanenzen in der Planungsregion Havelland-Fläming 2002/2003 im Rahmen der Erstellung von technischer Infrastruktur auftraten. Lediglich Eck et al. (2012) konnten in den sächsischen Kommunen 2009 keine Kostenremanenzen nachweisen, wobei jedoch die methodische Vorgehensweise der reinen Querschnittsbetrachtung methodisch diskussionswürdig erscheint, da diese innerhalb eines Zeitverlaufs und nicht eines einzelnen Zeitpunktes beobachtet werden können. Auch international wurden die Effekte des demografischen Wandels auf die öffentlichen Haushalte untersucht: Sowohl Grob und Wolter (2005) als auch Borge und Rattsø (1995) wiesen auf den Bildungsbereich bezogene Kostenremanenzeffekte in den Kantonen der Schweiz 1990-2002 bzw. den norwegischen Kommunen 1986-1989 nach. Auch am Beispiel der US-Staaten wurden empirische Untersuchungen durchgeführt, die sich aber vor allem auf die Altersstruktur konzentrierten und in denen darüber hinaus keine einheitlichen Ergebnisse erzielt wurden: Poterba (1996) stellte einen Rückgang der Ausgaben für Grundschulen je Grundschüler im Falle eines ansteigenden Anteils der über 65-Jährigen fest, Fernandez und Rogerson (2001) beschreiben einen Rückgang der Bildungsausgaben je Schüler bei einem steigenden Anteil der 5- bis 17-Jährigen bzw. Schülern insgesamt. Ladd und Murray (2001) wiesen ähnliche Regressionsergebnisse nach. In einer Stichprobe von Staaten aller Kontinente wies Kelley (1976) nach, dass die Staatsquote bei einem Anstieg des Altenquotienten (über 65-Jährige im Verhältnis zur erwerbstätigen Bevölkerung) ansteigt, was auf die betrachtete föderale Ebene (oberste Staatsebenen) zurückgeführt werden kann. Auf ein ähnliches Resultat kommt Zokalj (2016) in einer aktuelleren Studie über die 25 EU-Staaten.

\subsection{Altersstrukturkostenprofile}

Die Verschiebung der Altersstruktur verändert das Nachfrageverhalten der Bürger nach öffentlichen Leistungen, was wiederum die Ausgaben innerhalb des kommunalen Leistungskatalogs beeinflusst. Ein Großteil der kommunalen Aufgaben wird für spezifische Bevölkerungsaltersgruppen und somit altersklassenspezifisch erbracht. Sinkt beispielsweise der Anteil an unter 18-Jährigen, dann sinkt auch die Nachfrage nach kinder- und jugendspezifischen Einrichtungen, während hingegen ein Anstieg 
der Quote über 65-Jähriger den Bedarf an Einrichtungen der Pflegeinfrastruktur erhöht. Eine Vielzahl von Aufgaben wird demzufolge für spezifische Altersklassen wahrgenommen, weshalb von einer alterskohortenbezogenen Ausgaben- und Einnahmenstruktur in den Gebietskörperschaften ausgegangen werden kann. Mit Blick auf die kommunalen Ausgabenbelastungen ist dabei entscheidend, welche Alterskohorten einzelne kommunale Aufgabenbereiche besonders beanspruchen.

Die Altersstruktureffekte werden nachfolgend auf Basis der Arbeiten von Seitz (2007, S. $137 \mathrm{ff}$.) und Freigang (2012, S. 49f.) mittels sog. Altersstrukturkostenprofile ermittelt, die auf der Annahme basieren, dass verschiedene Alterskohorten unterschiedliche Pro-Kopf-Ausgaben bzw. -Zuschussbeträge in den öffentlichen Haushalten verursachen (ausführlicher Lenk et al. 2019a, S. 11; Seitz 2008, S. 49). Dabei wird betrachtet, welchen Anteil die jeweilige Altersgruppe an den kommunalen Einnahmen und Ausgaben hat. Anhand verschiedener statistischer Daten, bspw. dem Sozioökonomischen Panel oder der Einkommens- und Verbraucherstichprobe werden Indikatoren für die einzelnen Altersklassen ermittelt. Sofern der Einfluss einer Altersgruppe vernachlässigbar gering ist, erhält diese den Indikatorwert 0,0; die Altersgruppe, welche das höchste Pro-Kopf-Aufkommen aufweist, erhält den Wert 1,0. Analog wird für die Ausgabenseite vorgegangen. Aus der Schulstatistik ist z. B. die Altersstruktur der Schüler bekannt, sodass für Ausgaben im Schulbereich abgeleitet werden kann, in welcher Intensität von welcher Altersgruppe bestimmte Angebote in Anspruch genommen werden. Mittels empirischer Schätzung erfolgt dann die Ermittlung der Altersstrukturkostenprofile, welche die Höhe der Ausgaben je Einwohner pro Altersklasse anzeigen.

Mit Blick auf die bereinigten Ausgaben (Abb. 4a) wird deutlich, dass der höchste altersklassenbezogene Ausgabenanteil mit 7513€ je Einwohner auf die unter 6-Jährigen entfällt. Die zweithöchsten Pro-Kopf-Ausgaben finden sich in der Alterskohorte der 6- bis 18 -Jährigen (5404€/Einw.). Dies verdeutlicht, dass der Schwerpunkt der kommunalen Aufgabenerfüllung in der Betreuung der Kinder unter 6 Jahren liegt. Hinzu kommt, dass die Ausgaben je unter 6-Jährigem in 2016 durchschnittlich um 76\% höher als im Jahr 2000 liegen. Eine massive Ausgabensteigerung ergibt sich hierbei durch den seit August 2013 geltenden Rechtsanspruch auf eine frühkindliche Betreuung in einer Kindertagespflege oder Tageseinrichtung. Im Bereich der Alterskohorte von 6- bis 18-Jährigen, der ebenfalls ein deutliches Wachstum im Zeitablauf verzeichnet $(+69 \%)$, gilt vor allem das Schulwesen als Kostentreiber. Die Ausgaben im Bereich der über 65-Jährigen sind dagegen vergleichsweise niedrig, weil die nachgefragten Leistungen, wie Renten oder Leistungen aus der gesetzlichen Pflege- und Rentenversicherung, vorrangig durch den Bund bzw. die Sozialversicherung getragen werden und nicht durch die kommunale Ebene.

Die sich aus der Differenz der Ist-Ausgaben und zweckbezogenen Einnahmen einer Produktgruppe ergebenden Zuschussbeträge (Abb. 4b) geben Auskunft darüber, inwieweit die Kommunen die Leistungserbringung durch allgemeine Deckungsmittel (Steuereinnahmen und allgemeine Zuweisungen aus dem Kommunalen Finanzausgleich) finanzieren müssen. Da die Zuschussbeträge der unter 6-Jährigen und 6- bis 18-Jährigen positiv ausgeprägt sind, werden sie als Nettoempfänger öffentlicher Leistungen charakterisiert. Die durch die beiden Altersgruppen verursachten Ausgaben können durch die Einnahmen, die sie zur Finanzierung der kommunalen 
Abb. 4 Altersstrukturkostenprofile der Kommunen Mecklenburg-Vorpommerns 2000 und 2016 (Bereinigte Ausgaben a, Zuschussbeträge b). Quelle: Eigene Darstellung, eigene Berechnungen. Daten: Statistisches Amt Mecklenburg-Vorpommern
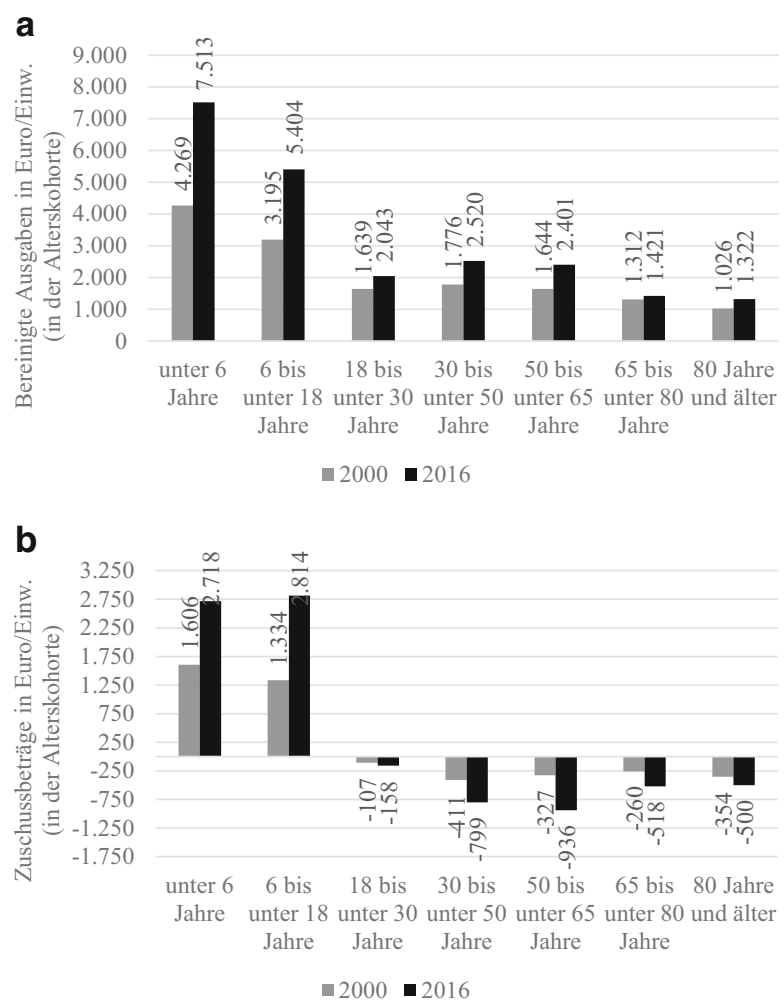

Haushalte beitragen, nicht gedeckt werden. Stattdessen müssen die Kommunen allgemeine Deckungsmittel einsetzen, um ihre Aufgaben im Zusammenhang mit der Kindertagesbetreuung und dem Schulwesen wahrzunehmen. Im Gegensatz dazu verzeichnen die anderen Alterskohorten negative Zuschussbeträge, d.h., die durch sie erbrachten Einnahmen übertreffen die verursachten Ausgaben, weshalb sie auch als Nettozahler kommunaler Leistungen bezeichnet werden. Die übrigen Altersklassen müssen demnach für die Inanspruchnahme von kommunalen Leistungen durch die unter 18-Jährigen aufkommen.

Dies ließe den Schluss zu, dass die Kommunen durch eine forcierte Verschiebung der Altersstruktur von der, ausgabenintensiven“ Altersgruppe der Kinder und Jugendlichen hin zu der, ertragreicheren“ Altersgruppe älterer Menschen aus fiskalischer Sicht profitieren könnten. Aus dem Zusammenspiel der Altersstrukturkostenprofile und der Entwicklung der Altersstruktur im Zeitverlauf (Abb. 2) sind jedoch Kostenremanenzeffekte zu vermuten, die dieser These entgegenstehen: So ist der Anteil der 6- bis 18-Jährigen deutlich abgesunken, während sich die Zuschussbeträge je 6- bis 18-Jährigen mehr als verdoppelt haben. Folglich ist von einem abnehmendem Auslastungsgrad schulischer Infrastruktur auszugehen, der die höheren Pro-Kopf-Ausgaben und -Zuschussbeträge erklärt. Der Anteil unter 6-Jähriger ist zwar angestiegen, die Ausgaben und Zuschussbeträge je unter 6-Jährigem haben sich aber jeweils fast verdoppelt. Ausgabensteigernde Faktoren sind hier zum einen 
der Rechtsanspruch auf frühkindliche Förderung seit 2013 und zum anderen die Notwendigkeit des Ausbaus von Kinderbetreuungs- und Schulinfrastruktur in wachsenden Städten, wie Rostock und Greifswald. Überdies sind die Verbesserungen beim Personalschlüssel und die massiven Tarifsteigerungen beim Personal im Sozial- und Erziehungsdienst als kostensteigernde Effekte zu bewerten (ausführlicher Lenk et al. 2019a, S. 89). Überdies kann ein sinkender Anteil an junger Bevölkerung mittelfristig auch in einem Verlust an erwerbsfähiger Bevölkerung resultieren, die am meisten zur Finanzierung der kommunalen Leistungserstellung beiträgt. Dies wirkt sich wiederum negativ auf die finanzielle Tragfähigkeit einer Gemeinde aus.

\section{Empirische Untersuchungen}

Auf Basis der zuvor beschriebenen Effekte wird nachfolgend der den regressionsanalytischen Berechnungen zugrundeliegende Datensatz beschrieben und eingeordnet (Abschn. 4.1). Mittels einer Fixed Effects-Regressionsanalyse wird daran anschlieBend untersucht, welche Effekte der Bevölkerungsrückgang und die Verschiebung der Altersstruktur auf die gemeindlichen Haushalte in Mecklenburg-Vorpommern ausüben (Abschn. 4.2). Zusätzlich wird mit Hilfe einer geografisch gewichteten Regression geprüft, ob und wie stark die Effekte des demografischen Wandels von der topografischen Lage einer Gemeinde abhängen (Abschn. 4.3).

\subsection{Datensatz}

Auf Grundlage der föderalen Verwaltungs- und Aufgabenstruktur in Deutschland ist der Bezug auf ein einzelnes Land zulässig und zweckmäßig. Dabei ist darauf hinzuweisen, dass Mecklenburg-Vorpommern eine besondere Gebietsstruktur aufweist (wenige, große Landkreise, sehr viele Gemeinden mit geringer Einwohnerzahl) und daher besonders stark vom demografischen Wandel betroffen ist, sodass die Befunde nur schwer generalisierbar sind. Dies liegt zudem auch daran, dass sich die Aufgabenzuordnung zwischen Landes- und Kommunalebene je nach Bundesland mitunter deutlich voneinander unterscheidet (Lenk et al. 2021, S. 50 ff.). Würden folglich Kommunen unterschiedlicher Flächenländer miteinander verglichen, besteht das Risiko, dass Einmaleffekte und landesspezifische Regelungen die Resultate Verzerrungen und Inkonsistenzen hinsichtlich der Ergebnisse hervorrufen.

Die Datengrundlage für die empirischen Berechnungen bilden die kommunale Jahresrechnungsstatistik Mecklenburg-Vorpommerns und weitere tief gegliederte Fachstatistiken. Sämtliche Datenquellen stehen in einzelgemeindlicher Form für die Jahre 2000 bis 2016 zur Verfügung, was eine Querschnitt-Längsschnitt-Regressionsanalyse mit einer echten (und damit lückenlosen) Paneldatenstruktur ermöglicht. Die Berechnungen fokussieren sich auf die Ebene der Gemeindeaufgaben, die sich aus dem Gemeindeaufgabenanteil der kreisfreien Städte, den vier großen kreisangehörigen Städten und den übrigen kreisangehörigen Gemeinden ergibt. Auf Basis des verwendeten Gebietsstandes liegen somit 751 kreisangehörige Gemeinden und 2 kreisfreie Städte als Beobachtungseinheiten in 17 Jahresscheiben vor. Der Bezug der empirischen Analysen auf die Gemeinden und Städte garantiert dabei den am 
tiefsten gegliederten Einblick in die kommunale Ebene. Zum anderen sind diese aufgrund der Wahrnehmung der Kindertagesbetreuung und der Schulträgeraufgaben besonders stark vom demografischen Wandel betroffen.

Die zu erklärenden Variablen sind die bereinigten Ausgaben und die Zuschussbeträge (jeweils in Euro je Einwohner) sowohl der laufenden Verwaltungs- als auch der Investitionstätigkeit. Dabei erfolgt eine Beschränkung auf die Einzelpläne 0 bis 8 bzw. die Hauptproduktbereiche 1 bis 5, womit die allgemeinen Deckungsmittel (Steuereinnahmen, allgemeine Zuweisungen und sonstige, zweckungebundene Zuweisungen und Zuwendungen) nicht in den zu erklärenden Variablen enthalten sind. Damit kann bestimmt werden, ob Bedarfstatbestände des demografischen Wandels aufgrund von Kostenremanenzen und Altersstruktureffekten vorrangig durch die allgemeinen Deckungsmittel und dabei insbesondere die Schlüsselzuweisungen aus dem Kommunalen Finanzausgleich finanziert werden müssen.

Die Zielstellung des Vergleichs der beiden Regressanden besteht darin, herauszufinden, ob die demografischen Einflussfaktoren unterschiedlich auf Ausgaben und Zuschussbeträge einwirken. Wirkt sich der Rückgang der Bevölkerung etwa nur auf die Ausgaben, nicht aber auf die Zuschussbeträge statistisch signifikant aus, so sollten demografische Bedarfstatbestände durch zweckgebundene Zuweisungen aufgefangen werden und bei der Finanzbedarfsermittlung innerhalb des Schlüsselzuweisungssystems nicht berücksichtigt werden. Ist die Wirkung hingegen bei beiden zu erklärenden Variablen ähnlich stark ausgeprägt, spricht dies für eine Berücksichtigung im System der Schlüsselzuweisungen (Kap. 5).

Die statistischen Vorbetrachtungen ergaben zwar, dass weder die Pro-Kopf-Zuschussbeträge noch die Pro-Kopf-Ausgaben normalverteilt sind (Jarque-Bera-Test, Shapiro-Wilk-Test und Kolmogorow-Smirnow-Anpassungstest); dies ist allerdings für die empirischen Betrachtungen unproblematisch, da die OLS-Schätzer bei derart großen Stichproben asymptotisch normalverteilt sind (Auer und Rottmann 2015, S. 460). Ohnehin handelt es sich bei dem Datensatz um eine Vollerhebung der Städte und Gemeinden Mecklenburg-Vorpommerns und nicht um eine Stichprobe. Demnach sind auch Ausreißerwerte typisch, die jedoch im weiteren Vorgehen nicht eliminiert werden, da damit ein aus inhaltlicher Sicht nicht nachvollziehbarer Informationsverlust einhergehen würde. Gemäß dem wissenschaftlichen Standard wurden beide zu erklärende Variablen logarithmiert, was die Interpretation der demografischen Effekte erleichtert und damit deutliche Vorteile mit sich bringt. Zudem wird vermutet, dass ein nicht-linearer Zusammenhang zwischen den beiden zu erklärenden Variablen und den Regressoren besteht (Abb. 3), der infolge einer Logarithmierung sachgerechter geschätzt werden kann. Die Logarithmierung der ProKopf-Zuschussbeträge verringert die Anzahl der Beobachtungseinheiten geringfügig, da einige Gemeinden im Zeitablauf negative Zuschussbeträge verzeichneten. Aus finanzwissenschaftlicher Perspektive kann zudem argumentiert werden, dass Gemeinden mit negativen Zuschussbeträgen erhebliche Überschüsse verzeichnen, ohne dass bereits die Einnahmen aus allgemeinen Deckungsmitteln hinzugerechnet wurden. Demnach wäre es grundsätzlich sachgerecht, diese kommunalen Einheiten aus den regressionsstatistischen Untersuchungen auszuschließen.

Auf diesen Überlegungen aufbauend werden die deskriptiven Kennzahlen aller Variablen in Tab. 2 dargestellt: 
Tab. 2 Deskriptive Statistik im Durchschnitt 2000-2016

\begin{tabular}{llllll}
\hline Variable & Mittelwert & Minimum & Maximum & $\begin{array}{l}\text { Standard- } \\
\text { abweichung }\end{array}$ & $N$ \\
\hline Zusch_EW & 451,09 & $-5284,67$ & $21.415,19$ & 342,39 & 12.801 \\
Ln_Zusch_EW & 5,53 & $-2,55$ & 9,97 & 1,60 & 11.898 \\
Ausg_EW & 1160,13 & $-863,08$ & $27.574,71$ & 694,89 & 12.801 \\
Ln_Ausg_EW & 6,95 & 3,75 & 10,22 & 0,36 & 12.799 \\
EWZahl_WR_1J & $99,09 \%$ & $39,19 \%$ & $169,82 \%$ & $2,78 \%$ & 12.048 \\
Q_u6 & $7,09 \%$ & $0,00 \%$ & $29,00 \%$ & $1,94 \%$ & 12.801 \\
Q_6-18 & $15,76 \%$ & $3,35 \%$ & $44,58 \%$ & $4,69 \%$ & 12.801 \\
Q_ü65 & $31,47 \%$ & $4,66 \%$ & $76,43 \%$ & $8,96 \%$ & 12.801 \\
AllgDM_EW & 711,88 & $-2736,28$ & $28.513,87$ & 457,40 & 12.801 \\
Ln_AllgDM_EW & 6,39 & 3,94 & 10,26 & 0,31 & 12.794 \\
\hline
\end{tabular}

Quelle: Eigene Darstellung, eigene Berechnungen. Daten: Statistisches Amt Mecklenburg-Vorpommern (Haushaltsrechnungsstatistik, Kernhaushalte).

- In_Zusch_EW, In_Ausg_EW: Die Resultate hinsichtlich der bereinigten Ausgaben bieten eine Vergleichsmöglichkeit zu bereits veröffentlichten Studien zu Kostenremanenzeffekten. Die Zuschussbeträge sind dagegen besonders relevant für Sonderbedarfstatbestände, die den Kommunen im Schlüsselzuweisungssystem anerkannt werden sollten.

- EWZahl_WR: Die Ein-Jahres-Wachstumsrate der Einwohnerzahl repräsentiert ein zentrales Element der nachfolgenden Untersuchungen. Wichtig ist hierbei, dass es sich um eine dynamische Wachstumsrate handelt: So wird die Veränderung der Einwohnerzahl im Jahr 2016 gegenüber 2015, 2015 gegenüber 2014 etc. gemessen. Dies bewirkt eine Verringerung der Anzahl an Beobachtungen, da die Einwohnerzahlen vor dem Jahr 2000 nicht zur Verfügung stehen. Die Wachstumsraten der Einwohnerzahlen werden aufgrund der Vermutung eines nicht-linearen Zusammenhangs logarithmiert.

- $Q \_$6, $Q \_6-18 \_E W, Q \_\ddot{u} 65$ : Die Rolle der Altersstruktur soll mittels der Anteile der unter 6-Jährigen, 6- bis 18-Jährigen und über 65-Jährigen in die Regressionsanalysen eingehen. In Anlehnung an das Konzept von Jugend- und Altenquotienten (Starke 2020, S. $170 \mathrm{ff}$.) wird die Anzahl der Personen im erwerbsfähigen Alter (19-64 Jahre) als Relationsmaßstab verwendet. Dies hat den Vorteil, dass die Alterskohorten, welche die Zuschussbeträge verursachen, direkt den Altersgruppen gegenübergestellt werden, welche hauptsächlich die Steuereinnahmen erwirtschaften.

- In_AllgDM_EW: Mittels dieser Kontrollvariable soll ein selbst verstärkender Effekt von Gemeinden mit einem überdurchschnittlich hohen Bestand an allgemeinen Deckungsmitteln (Steuereinnahmen und allgemeine Zuweisungen) überprüft werden. Würde diese Variable nicht einbezogen, dann könnten interkommunale relative Bedarfsunterschiede falsch bewertet und zu Ungunsten von Gemeinden mit einem geringen Bestand an Deckungsmitteln verzerrt werden. Im Sinne einer besseren Interpretation wird die Variable logarithmiert. 


\subsection{Fixed Effects-Analyse}

Um die zeitliche Komponente des Datensatzes adäquat abzubilden, wird nachfolgend ein Fixed Effects-Regressionsmodell gemäß der Formel

$$
\ln \left(y_{i, t}\right)=\alpha_{i}+\ln \left(y_{i, t-1}\right) \gamma+x^{\prime}{ }_{i, t} \beta+\varepsilon_{i, t}
$$

geschätzt, welches die Entwicklung der Variablen je Beobachtungseinheit ausschließlich über die Zeit (Längsschnitt) interpretiert. Die Bestandteile der Formel werden dabei wie folgt definiert:

- Der Term $\ln \left(\mathrm{y}_{\mathrm{i}, \mathrm{t}}\right)$ enthält die endogene(n) Variable(n), d.h., die logarithmierten bereinigten Ausgaben bzw. Zuschussbeträgen in Euro je Einwohnern.

- Der K×1-Vektor $\mathbf{x}^{\prime}$ beinhaltet die exogenen Variablen, für die ein (i.d.R. linearer) Zusammenhang mit dem Regressanden unterstellt wird.

- Mittels des Terms $\alpha$ wird die im Rahmen der empirischen Berechnungen ermittelte Konstante wiedergegeben. Sie gibt im Regelfall die Höhe der Ist-Ausgaben bzw. Zuschussbeträge an, die ohne den Einfluss der erklärenden Variablen gemessen wird.

- Die Regressionskoeffizienten, die jeweils den Einfluss einer erklärenden Variablen auf die Ist-Ausgaben bzw. Zuschussbeträge messen, werden durch den Term $\beta$ in der Regressionsgleichung abgebildet und repräsentieren die wichtigste Maßzahl in den nachfolgenden Analysen.

- Darüber hinaus stellt der Term $\mathrm{y}_{\mathrm{i}, \mathrm{t}-1}$ den verzögerten Regressanden dar, $\gamma$ ist der zugehörige Regressionskoeffizient. Damit soll der grundsätzlich positive Trend der Pro-Kopf-Ausgaben und -Zuschussbeträge über die Zeit, der durch eine allgemeine Preissteigerung, aber auch infolge von Aufgabenübertragungen durch das Land verursacht sein könnte, nachvollzogen werden.

- Der Term $\varepsilon_{i, t}$ bildet die Störgröße bzw. statistische Restgröße der empirischen Schätzung ab. Er beinhaltet dabei sämtliche Effekte, welche nicht von den im Vektor $\mathbf{x}^{\prime}$ erfassten Variablen ausgehen, sondern von anderen - innerhalb des zugrundliegenden Modells nicht einbezogenen - Variablen stammen.

Es werden einerseits Modelle geschätzt, die sich auf den Ist-Stand der Ausgaben und Zuschussbeträge beziehen. Damit wird spezifiziert, dass die Ausprägungen der jeweiligen Regressionskoeffizienten vom Zeitpunkt und der Gemeinde selbst abhängig sein können. Jedoch erscheint es problematisch, dass lediglich die Entwicklung der Einwohnerzahl als Wachstumsrate dargestellt wird, während die übrigen Variablen einen Ist-Stand repräsentieren. Aus diesem Grund werden zusätzlich die logarithmierten Wachstumsraten der bereinigten Pro-Kopf-Ausgaben und -Zuschussbeträge den logarithmierten Ein-Jahres-Wachstumsraten aller Regressoren (gekennzeichnet mit Präfix ln_und Suffix _WR) gegenübergestellt. In der Folge werden die Entwicklungen der Variablen über die Zeit miteinander verglichen, wodurch insbesondere die Effekte des demografischen Wandels besser zu erklären sind, als 
über den Ist-Stand von Ausgaben und Zuschussbeträgen. Die veränderte Regressionsformel stellt sich wie folgt dar:

$$
\ln \left(\frac{y_{i, t}}{y_{i, t-1}}\right)=\alpha_{i}+\ln \left(\frac{y_{i, t-1}}{y_{i, t-2}}\right) \gamma+\ln \left(\frac{x^{\prime}{ }_{i, t-1}}{x^{\prime}{ }_{i, t-2}}\right) \beta+\frac{\varepsilon_{i, t}}{\varepsilon_{i, t-1}} .
$$

Tab. 3 zeigt die Modellergebnisse aller geschätzten Regressionsmodelle, die in ihrer Gesamtheit allesamt statistisch signifikant sind, was die durchweg hohen F-Statistiken belegen. Die Bestimmtheitsmaße sind darüber hinaus insgesamt niedrig bis moderat ausgeprägt; höhere Ausprägungen ergeben sich bei den Modellen, die ausschließlich Wachstumsraten verwenden.

Dabei wirkt sich die Ein-Jahres-Wachstumsrate der Einwohnerzahl negativ (und dabei statistisch signifikant auf mindestens dem 5\%-Niveau) sowohl auf den IstStand als auch die Wachstumsrate der Pro-Kopf-Zuschussbeträge und -Ausgaben aus. Entsprechend der in Abb. 3 vorgenommenen Klassifizierung ist dies in Bezug auf die Ist-Stände als Zeichen für milde Kostenremanenzen zu interpretieren: Ein

Tab. 3 Fixed Effects-Regressionsanalyse 2000-2016

\begin{tabular}{|c|c|c|c|c|}
\hline \multirow[t]{2}{*}{ Variable } & \multicolumn{2}{|c|}{ Zuschussbeträge je Einwohner } & \multicolumn{2}{|c|}{ Bereinigte Ausgaben je Einwohner } \\
\hline & $\begin{array}{l}\text { Ist-Stand } \\
\text { (ln_Zusch_EW) }\end{array}$ & $\begin{array}{l}\text { Wachstumsraten } \\
\text { (ln_Zusch_EW_WR) }\end{array}$ & $\begin{array}{l}\text { Ist-Stand } \\
\text { (ln_Ausg_EW) }\end{array}$ & $\begin{array}{l}\text { Wachstumsraten } \\
\text { (ln_Ausg_EW_WR) }\end{array}$ \\
\hline (Konstante) & $\begin{array}{l}2,3945 * * * \\
(0,2842)\end{array}$ & $\begin{array}{l}0,0236 * * * \\
(0,0039)\end{array}$ & $\begin{array}{l}3,1195 * * * \\
(0,1593)\end{array}$ & $\begin{array}{l}0,0188 * * * \\
(0,0016)\end{array}$ \\
\hline $\begin{array}{l}\text { L1_ln_Zusch_EW } \\
(\text { _WR })\end{array}$ & $\begin{array}{l}0,0588 * * * \\
(0,0136)\end{array}$ & $\begin{array}{l}-0,4549 * * * \\
(0,0117)\end{array}$ & - & - \\
\hline $\begin{array}{l}\text { L1_ln_Ausg_EW } \\
\text { (_WR) }\end{array}$ & - & - & $\begin{array}{l}0,2902 * * * \\
(0,0181)\end{array}$ & $\begin{array}{l}-0,3977 * * * \\
(0,0121)\end{array}$ \\
\hline Ln_EWZahl_WR_1J & $\begin{array}{l}-0,4484^{* *} \\
(0,2178)\end{array}$ & $\begin{array}{l}-0,9059 * * \\
(0,2893)\end{array}$ & $\begin{array}{l}-0,4821 * * * \\
(0,0980)\end{array}$ & $\begin{array}{l}-1,1826^{* * * *} \\
(0,1193)\end{array}$ \\
\hline$\left(\mathrm{ln} \_\right)$Q_u6 (_WR) & $\begin{array}{l}1,4104 * * * \\
(0,5264)\end{array}$ & $\begin{array}{l}0,0587 \\
(0,0544)\end{array}$ & $\begin{array}{l}0,5476 * * * \\
(0,1919)\end{array}$ & $\begin{array}{l}-0,0117 \\
(0,0189)\end{array}$ \\
\hline $\begin{array}{l}\left(\ln \_\right) \text {Q_6-18 } \\
(\text { (WR) }\end{array}$ & $\begin{array}{l}0,6556 * * * \\
(0,2279)\end{array}$ & $\begin{array}{l}0,2991 * * * \\
(0,1078)\end{array}$ & $\begin{array}{l}-0,1342 \\
(0,0904)\end{array}$ & $\begin{array}{l}0,1289 * * * \\
(0,0281)\end{array}$ \\
\hline$\left(\ln \_\right)$Q_ü65 (_WR) & $\begin{array}{l}-0,3531 * \\
(0,1944)\end{array}$ & $\begin{array}{l}-0,2819 * * \\
(0,1247)\end{array}$ & $\begin{array}{l}0,2118 * * * \\
(0,0765)\end{array}$ & $\begin{array}{l}-0,1771 * * * \\
(0,0487)\end{array}$ \\
\hline $\begin{array}{l}\text { Ln_AllgDM_EW } \\
(\text { _WR })\end{array}$ & $\begin{array}{l}0,4252 * * * \\
(0,0444)\end{array}$ & $\begin{array}{l}0,0896 * * * \\
(0,0452)\end{array}$ & $\begin{array}{l}0,2712 * * * \\
(0,0247)\end{array}$ & $\begin{array}{l}-0,0119 \\
(0,0323)\end{array}$ \\
\hline$N$ & 10.617 & 9654 & 12.038 & 11.278 \\
\hline $\mathrm{R}^{2}$ (within) & 0,0367 & 0,2262 & 0,2197 & 0,1701 \\
\hline $\mathrm{R}^{2}$ (between) & 0,0390 & 0,0848 & 0,0018 & 0,0780 \\
\hline $\mathrm{R}^{2}$ (overall) & 0,0078 & 0,2177 & 0,0120 & 0,1644 \\
\hline F-Statistik & 21,54 & 269,56 & 116,24 & 215,17 \\
\hline
\end{tabular}

Standardfehler in Klammern.

* ... statistisch signifikant auf dem $10 \%$-Niveau,

** ... statistisch signifikant auf dem $5 \%$-Niveau,

*** ... statistisch signifikant auf dem $1 \%$-Niveau

Quelle: Eigene Darstellung, eigene Berechnungen. Daten: Statistisches Amt Mecklenburg-Vorpommern (Haushaltsrechnungsstatistik, Kernhaushalte). 
Absinken der Einwohnerzahl um 10 Prozentpunkte führt demnach im Durchschnitt c. p. zu einem um 4,5\% (4,8\%) höheren Niveau der Pro-Kopf-Zuschussbeträge (ProKopf-Ausgaben). Werden statt den Ist-Ständen die Wachstumsraten der zu erklärenden Variablen herangezogen, sind im Falle der Ausgaben sogar erhebliche Kostenremanenzen nachweisbar: Sinkt die Einwohnerzahl demnach um 10\%, dann steigen die Pro-Kopf-Zuschussbeträge (Pro-Kopf-Ausgaben) c.p. durchschnittlich um 9,1\% $(11,8 \%)$. Somit existieren zwar durchaus kleinere Unterschiede in der Ausprägung der Remanenzeffekte, je nachdem, ob Zuschussbeträge oder Ausgaben bzw. IstStände oder Wachstumsraten untersucht werden; die Wirkrichtung der Ergebnisse ist allerdings die gleiche. Wichtig ist dabei, dass der Effekt bei den bereinigten Ausgaben stärker ausfällt als bei den Zuschussbeträgen. Dies deutet darauf hin, dass die Gemeinden einen Teil der Kostenremanenzen bereits über zweckgebundene Einnahmen oder Fördermittel abdämpfen können. Der durch die Zuschussbeträge angezeigte Teil der Remanenzen kann wiederum mittels eines Nebenansatzes bei der Bestimmung des Finanzbedarfs im Schlüsselzuweisungssystem aufgefangen werden.

Mit Blick auf die Altersstruktur erscheint vorrangig der Modellansatz mit Bezug auf die Ist-Stände der verwendeten Variablen geeignet, die durch die Altersstrukturkostenprofile vermuteten Zusammenhänge adäquat abzubilden: Eine um 10 Prozentpunkte höhere Quote sowohl an unter 6-Jährigen als auch 6- bis 18-Jährigen in Relation zur potenziell erwerbsfähigen Bevölkerung bewirkt c.p. eine durchschnittliche (statistisch hoch signifikante) Erhöhung der Pro-Kopf-Zuschussbeträge um 14,1\% bzw. 6,6\%. Bezugnehmend auf die Ausgaben fallen die Koeffizienten betraglich geringer aus und sind zudem bei den 6- bis 18-Jährigen statistisch nicht signifikant. Wichtig ist hierbei, dass die Förderung von Kindern in Tageseinrichtungen und in Tagespflege eine Gemeindeaufgabe darstellt, was den hohen positiven Koeffizienten eine kausale Grundlage gibt. Der im Vergleich niedrigere Regressionskoeffizient des Anteils der 6- bis 18-Jährigen ist auf die Rolle der Umlage an die Schulträgerkommunen in Mecklenburg-Vorpommern zurückzuführen: Weiterführende, ausgabenintensive weiterführende Schulen sind i.d.R. nicht Teil der Kreisaufgaben und nicht der in den Regressionsmodellen betrachteten Gemeindeaufgaben, wodurch sie außerhalb der Erklärungskraft des Regressionsmodells liegen. Der Anteil der über 65-Jährigen wirkt sich negativ (positiv) und zumindest auf dem 10\%-Niveau statistisch signifikant auf die Zuschussbeträge (Ausgaben) aus, wodurch sich kein eindeutiges Bild ergibt. Dies ändert sich, wenn die Variablen vollständig als Wachstumsraten in das Fixed Effects-Modell eingehen: In dem Fall bewirkt eine 10-prozentige Erhöhung der Quote an über 65-Jährigen c. p. eine durchschnittliche Absenkung der Pro-KopfZuschussbeträge (Pro-Kopf-Ausgaben) um 2,8\% (1,8\%). Im Unterschied dazu sinkt der Erklärungsgehalt vom Einfluss der Quoten an unter 6-Jährigen und 6- bis 18Jährigen: So ist erstere in Bezug auf beide zu erklärende Variablen insignifikant, wofür möglicherweise die im Zeitraum stattgefundene leicht positive Entwicklung des Anteils der Kinder unter 6 Jahren verantwortlich ist (Abb. 2). Demnach ist hier nicht die Wachstumsrate für die Entwicklung der Zuschussbeträge bzw. bereinigten Ausgaben im Zeitverlauf ausschlaggebend. Stattdessen ist der Ist-Stand der Quote für die Quantifizierung der kommunalen Lasten aus der Kinderbetreuung entscheidend für die Höhe der kommunalen Ausgabenlasten. Der Anteil 6- bis 18-Jähriger wirkt dagegen sowohl auf die Ausgaben als auch die Zuschussbeträge positiv ein. 
Tab. 4 Gegenüberstellung der Effekte von Bevölkerungsrückgang und Alterung auf Basis der Ergebnisse des Fixed Effects-Modells mit Wachstumsraten

\begin{tabular}{|c|c|c|c|c|}
\hline & \multicolumn{2}{|c|}{ Zuschussbeträge } & \multicolumn{2}{|c|}{ Bereinigte Ausgaben } \\
\hline & $\begin{array}{l}\text { Einwohner- } \\
\text { zahl }\end{array}$ & 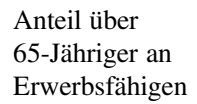 & $\begin{array}{l}\text { Einwohner- } \\
\text { zahl }\end{array}$ & $\begin{array}{l}\text { Anteil über } \\
\text { 65-Jähriger an } \\
\text { Erwerbsfähigen }\end{array}$ \\
\hline $\begin{array}{l}\text { Ist-Entwicklung 2000-2016 (Loga- } \\
\text { rithmierte Wachstumsrate) }\end{array}$ & $97,54 \%$ & $166,84 \%$ & $97,54 \%$ & $166,84 \%$ \\
\hline $\begin{array}{l}\text { Regressionskoeffizient (Fixed Ef- } \\
\text { fects-Modell) }\end{array}$ & $-0,9059$ & $-0,2819$ & $-1,1826$ & $-0,1771$ \\
\hline Effekt über den Gesamtzeitraum in \% & $+88,37 \%$ & $-47,03 \%$ & $+115,36 \%$ & $-29,55 \%$ \\
\hline
\end{tabular}

Quelle: Eigene Darstellung, eigene Berechnungen. Daten: Statistisches Amt Mecklenburg-Vorpommern (Haushaltsrechnungsstatistik, Kernhaushalte)

Hier ist zu beachten, dass diese Quote im Zeitverlauf deutlich abgenommen hat. Der Zusammenhang ist deshalb so zu interpretieren, dass der absinkende Anteil dieser Altersgruppe auch zu im Zeitverlauf niedrigeren Zuschussbeträgen bzw. Ausgaben je Einwohner geführt hat.

Die Höhe bzw. Wachstumsrate der allgemeinen Deckungsmittel wirkt sich indes positiv auf die Pro-Kopf-Zuschussbeträge und -Ausgaben aus. Dies kann damit begründet werden, dass Gemeinden mit mehr frei verfügbaren finanziellen Mitteln tendenziell auch mehr Ausgaben tätigen. Dieser sog. Leuchtturmeffekt wird mittels der Variable entsprechend aufgefangen, wodurch die anderen Regressionskoeffizienten nicht verzerrt werden.

In der Gesamtschau fällt auf, dass der Bevölkerungsrückgang im Durchschnitt zu steigenden Zuschussbeträgen bzw. Ausgaben je Einwohner führt. Dagegen resultiert eine Erhöhung des Anteils der über 65-jährigen Bevölkerung in einer Absenkung von Zuschussbeträgen und Ausgaben. Anhand der Ist-Entwicklung der Einwohnerzahl und der Quote an über 65-Jährigen im Zeitraum von 2000 bis 2016 in Kombination mit den Regressionskoeffizienten (in Bezug auf Wachstumsraten) soll nun näherungsweise abgeleitet werden, welcher der beiden Effekte im Zeitverlauf stärker war (Tab. 4). Dabei kann konstatiert werden, dass sich das Wachstum der Pro-KopfZuschussbeträge allein durch den Effekt der absinkenden Einwohnerzahl im Zeitraum von 2000-2016 um mehr als 88\% erhöhte. Zwar fiel die Ist-Wachstumsrate des Anteils der über 65-Jährigen im Vergleich zum Bevölkerungsrückgang höher aus; der Regressionskoeffizient ist im Betrag jedoch deutlich niedriger. Infolgedessen trägt der steigende Anteil der Alterskohorte dazu bei, dass das Wachstum der Pro-Kopf-Zuschussbeträge um $47 \%$ niedriger ausfiel. Der Einfluss der sinkenden Einwohnerzahl im Verhältnis zur Alterung der Bevölkerung ist somit aus quantitativer Sicht deutlich stärker ausgeprägt, wobei sich diese Resultate auch mit Blick auf die bereinigten Ausgaben bestätigen und die Effekte sogar noch verstärken.

\subsection{Geografisch gewichtete Regression}

Während im vorhergehenden Abschnitt vorrangig die zeitlichen Effekte des demografischen Wandels analysiert wurden, soll nachfolgend geprüft werden, ob zudem erhebliche räumliche Unterschiede im Hinblick auf die nachgewiesenen Kostenre- 
Abb. 5 Grafische Darstellung der räumlichen $\mathrm{Ge}$ wichtung von Datenpunkten um den Regressionspunkt. r Regressionspunkt, qi umliegender Datenpunkt, $w_{\mathrm{ij}}$ Gewichtung der Datenpunkte, $\mathrm{d}_{\mathrm{ij}}$ Distanz zwischen Regressions- und Datenpunkt. Quelle: Eigene Darstellung nach Fotheringham et al. 2010, S. 44

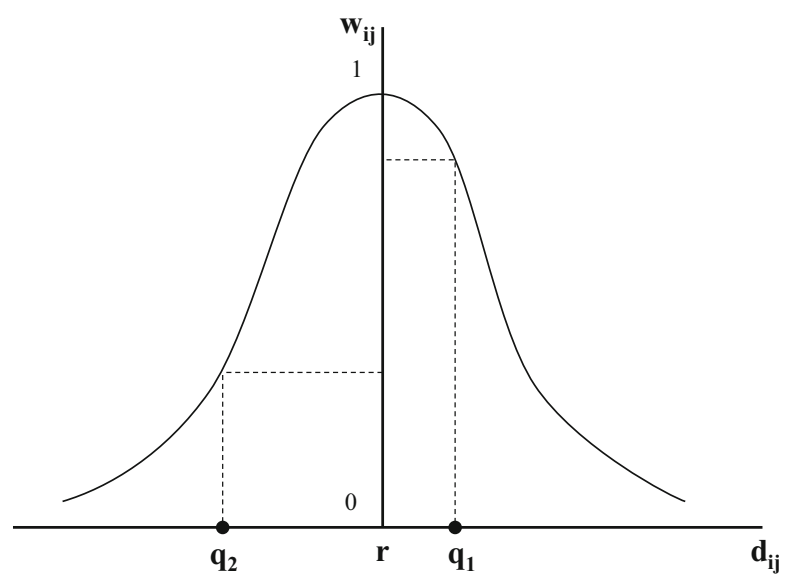

manenzen bestehen. Gerade zwischen Gemeinden sind räumliche Differenzen anzunehmen, da sie offene Systeme sind, die durch intraregionale Wirtschaftsströme miteinander verbunden sind (Zimmermann et al. 2017, S. 254f.). Basierend darauf haben sie aus topografischer, raumstruktureller und demografischer Sicht unterschiedliche Voraussetzungen, ihre Aufgaben sachgerecht zu erfüllen. Deshalb ist nicht davon auszugehen, dass die in den globalen Modellen nachgewiesenen Kostenremanenzen in allen Gemeinden den gleichen Koeffizienten aufweisen. Stattdessen könnte es in Mecklenburg-Vorpommerns Regionen geben, in denen die negativen Folgen des Bevölkerungsrückgangs geringer oder stärker ausfallen. Oftmals geht bspw. ein erheblicher Einfluss von der demografischen Lage in den Nachbargemeinden auf eine bestimmte Gemeinde aus, wodurch Abweichungen vom Durchschnitt zustande kommen. Deutliche Unterschiede in der Bevölkerungsentwicklung waren bereits in Abb. 1 sichtbar: Den stark schrumpfenden Gemeinden an der Grenze zwischen den Landkreisen Mecklenburgische Seenplatte und Vorpommern-Greifswald stehen tendenziell wachsende Gemeinden im Umfeld der Oberzentren gegenüber. Dabei ist insbesondere zu hinterfragen, ob auch die Kostenremanenzeffekte in diesen Gemeinden regional spezifischen Anpassungs- und Handlungsmustern folgen, für deren Nachweis sich die Methodik der geografisch gewichteten Regression (ausführlicher Fotheringham et al. 2010) primär eignet.

Zur Darstellung topografischer und geografischer Besonderheiten schätzt die Methode lokale Regressionskoeffizienten (ausführlicher Brunsdon et al. 1998, S. 433), die auf Karten visualisiert werden. Dafür wird für jede einzelne der 753 Gemeinden (in Abb. 5 Regressionspunkt r) eine OLS-Schätzung durchgeführt, wobei jede umliegende Gemeinde q entsprechend ihrer Entfernung gewichtet wird: Die näher liegende Gemeinde $\mathrm{q}_{1}$ wird höher und der weiter entfernte Punkt $\mathrm{q}_{2}$ geringer gewichtet (ausführlicher Eckey et al. 2007, S. 52; Fábián 2014, S. 62).

Der Aufbau des geografisch gewichteten Regressionsmodells folgt den bereits durchgeführten Analysen. Jedoch kann die zuvor etablierte Paneldatenstruktur für diese Methode softwaretechnisch nicht eingesetzt werden, sondern lediglich eine multivariate OLS-Regressionsanalyse für einen Zeitpunkt als Basis dienen. Deshalb werden nur die Pro-Kopf-Ausgaben und nicht die im Zeitverlauf stark schwankenden 


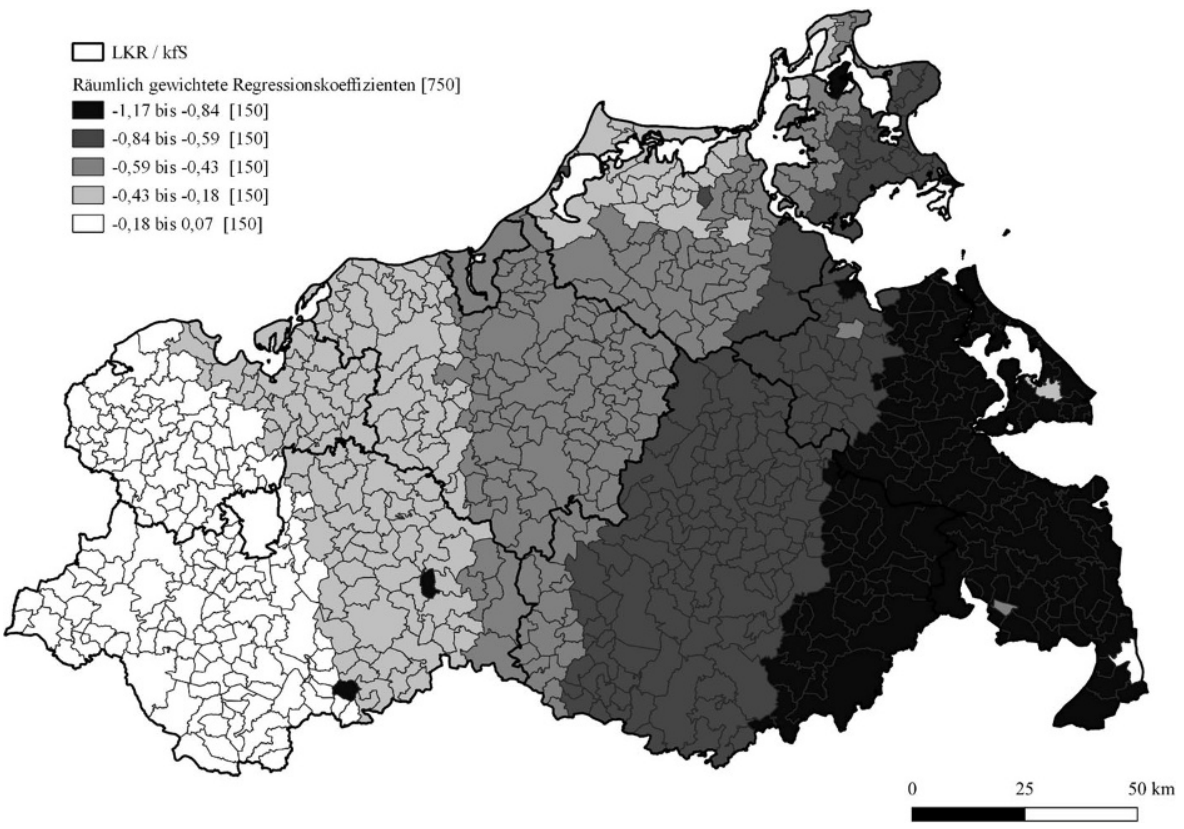

Abb. 6 Räumlich gewichtete Regressionskoeffizienten der Wachstumsrate der Einwohnerzahl in Bezug auf die Wachstumsrate der bereinigten Ausgaben je Einwohner 2016 ggü. 2000. Quelle: Eigene Darstellung, eigene Berechnungen. Daten: Statistisches Amt Mecklenburg-Vorpommern, Bevölkerungsstand jeweils zum 31.12., Herausgeber der Kartengrundlage: GeoBasis-DE/MV; dl-de/by-2-0

Pro-Kopf-Zuschussbeträge als zu erklärende Variable betrachtet. Dabei werden die Wachstumsraten der Pro-Kopf-Ausgaben und die Wachstumsraten der Einwohnerzahlen für das Jahr 2016 gegenüber 2000 als Variablen modelliert und deren Relation zueinander geschätzt. Die ermittelten Regressionsergebnisse erbringen gemäß des Signifikanztests auf räumliche Nicht-Stationarität einen statistischen Mehrwert gegenüber einer globalen Regressionsanalyse.

Abb. 6 illustriert eine deutliche topografische Variation der räumlich gewichteten Regressionskoeffizienten für die Wachstumsrate der Einwohnerzahl vom Westen in den Osten Mecklenburg-Vorpommerns. Dabei deuten dunkler eingefärbte Kommunen auf erhöhte Kostenremanenzen hin, während heller dargestellte Kommunen eine vergleichsweise geringe Ausgabensteigerung infolge eines Rückgangs ihrer Einwohner aufweisen. Speziell die östlich gelegenen Gemeinden, die den stärksten Bevölkerungsrückgang verzeichnen (siehe Abb. 1), weisen auch die stärksten Kostenremanenzeffekte auf. Mit Ausnahme Neubrandenburgs im Landkreis Mecklenburgische Seenplatte finden sich in der Umgebung keine Oberzentren, die Ausgabenanstieg in Gemeinden mit rückläufigen Einwohnerzahlen mittels der Erfüllung ihrer zentralörtlichen Funktionen auffangen könnten. Entsprechend belaufen sich die ermittelten lokalen Regressionskoeffizienten bis auf einen Wert i.H.v. $-1,17$, was auf erhebliche Kostenremanenzeffekte hindeutet.

Je näher die Gemeinden dabei zu den Oberzentren Greifswald und Stralsund im Norden sowie Rostock und Schwerin im Westen liegen, scheinen die Kostenrema- 
nenzen abzunehmen. Möglicherweise sparen die dort schrumpfenden Gemeinden Ausgaben ein, weil die Zentralen Orte Teile ihrer Aufgabenerfüllung schleichend übernehmen. Gemeinden in Verflechtungsbereichen können sich daher im Regelfall auf den nächstgelegenen Zentralen Ort verlassen, während die peripher gelegenen Gemeinden im Osten Mecklenburg-Vorpommerns die Leistungen selbst weiter erbringen müssen und deswegen höhere Kostenremanenzen verzeichnen. In Richtung der Westgrenze nehmen die Regressionskoeffizienten betraglich deutlich ab, was zugleich für geringere Ausgabensteigerungen im Falle eines Einwohnerrückgangs spricht. Entsprechend kann angenommen werden, dass sich die Grenznähe zu den im Vergleich finanzstärkeren Kommunen Niedersachsens (mit der Großstadt Lüneburg) und Schleswig-Holsteins (mit dem Oberzentrum Lübeck) sowie dem Stadtstaat Hamburg positiv auf die Entwicklung der Einwohnerzahlen auswirkt.

Die Grenznähe zu Brandenburg ist dagegen ambivalent zu beurteilen: Offensichtlich scheint die Nähe zum im Nordosten Brandenburgs gelegenen Landkreis Uckermark, der tendenziell finanz- und wirtschaftskraftschwächere Gemeinden umfasst, keine Entspannungseffekte hinsichtlich der Kostenremanenzen hervorzurufen. Westwärts nehmen die Regressionskoeffizienten im Betrag dagegen ab; dabei ist allerdings unklar, ob dies an der Grenznähe zu Niedersachsen, der geringeren Entfernung zu Schwerin oder der Nähe zu den brandenburgischen Landkreisen Prignitz bzw. Ostprignitz-Ruppin liegt.

Weiterhin ist erkennbar, dass die Landkreisgrenzen recht unbedeutend für die Stärke der Kostenremanenzen zu sein scheinen. Die Komponente der unterschiedlichen Vorgaben der Rechtsaufsicht scheint daher keine unmittelbare Rolle für die Folgen des demografischen Wandels zu spielen, da diese eine Kreisaufgabe darstellt.

Die Nähe zur Ostseeküste scheint in Bezug auf die Höhe der Kostenremanenzeffekte keinen dominanten Einfluss zu haben, da die Koeffizienten eher von Westen nach Osten schwanken. Auf der Insel Rügen sowie der Halbinsel Darß sind allerdings betraglich niedrigere Koeffizienten und damit weniger starke Remanenzeffekte zu beobachten. Ursächlich dafür ist der Einfluss der Tourismuswirtschaft, demzufolge sich die kommunalen Ausgaben nicht nur an der eigenen Einwohnerzahl, sondern auch der Anzahl der Touristen orientieren (Infrastruktur, Gewerbeamt, Ordnung und Sicherheit etc.).

In der Gesamtschau stellt dieser Ansatz einen wichtigen Schritt in der Erforschung von Kostenremanenzeffekten dar, da er die interkommunale Variation darstellt, die mit Hilfe eines globalen Regressionsmodells nicht abgebildet werden kann. Neben der Feststellung, dass peripher gelegene Gemeinden mit einem stärkeren Bevölkerungsrückgang deutlich größere Kostenremanenzeffekte aufweisen, ist auf den möglichen Einfluss angrenzender Länder sowie der Küstennähe auf die Stärke der Effekte hinzuweisen.

\section{Demografische Sonderbedarfe im Kommunalen Finanzausgleich}

Die empirischen Untersuchungen zeigen eindrücklich, dass der Rückgang der Bevölkerung und die Veränderung der Altersstruktur die kommunale Ebene vor erhebliche strukturelle Probleme bei der Wahrnehmung ihres Aufgabenkanons stellen. Um eine 
auskömmliche finanzielle Ausstattung auch in schrumpfenden Gemeinden bereitzustellen, bietet sich dabei insbesondere das Instrument des Kommunalen Finanzausgleichs an (Abschn. 5.1). Daher soll im Weiteren speziell auf die Berücksichtigung demografieinduzierter Finanzbedarfe im Schlüsselzuweisungssystem eingegangen werden (Abschn. 5.2). Abschließend soll geklärt werden, inwiefern sich die empirischen Ergebnisse auf die Gestaltung von Kommunalen Finanzausgleichssystemen auswirken (Abschn. 5.3).

\subsection{Funktionsweise}

Innerhalb des Kommunalen Finanzausgleichs wird zwischen zweckgebundenen Zuweisungen und von den Kommunen frei verwendbaren Schlüsselzuweisungen unterschieden (Abb. 7). Die Höhe der Schlüsselmasse einer Gemeinde wird auf Basis eines Vergleichs der individuellen Finanzkraft und des individuellen Finanzbedarfs bestimmt: Die Finanzkraft besteht im Regelfall aus den Gemeindeanteilen an Einkommen- und Umsatzsteuer sowie den nivellierten Realsteuereinnahmen.

Zur Bestimmung des Finanzbedarfs werden standardisierte Bedarfsgrößen gebildet, wobei ein auf der Einwohnergröße einer Kommune basierender Hauptansatz und ggf. eine bestimmte Zahl an Nebenansätzen zur Anwendung kommen. Die Summe dieser Ansätze entspricht dem nicht-monetären Gesamtansatz der Kommune. Dieser Ansatz wird mit dem sog. Grundbetrag multipliziert, wodurch ein rechnerischer Vergleich zwischen Finanzkraftmesszahl und Bedarfsmesszahl zustande kommt. Ist der individuelle Finanzbedarf einer Kommune höher als deren Finanzkraft, dann

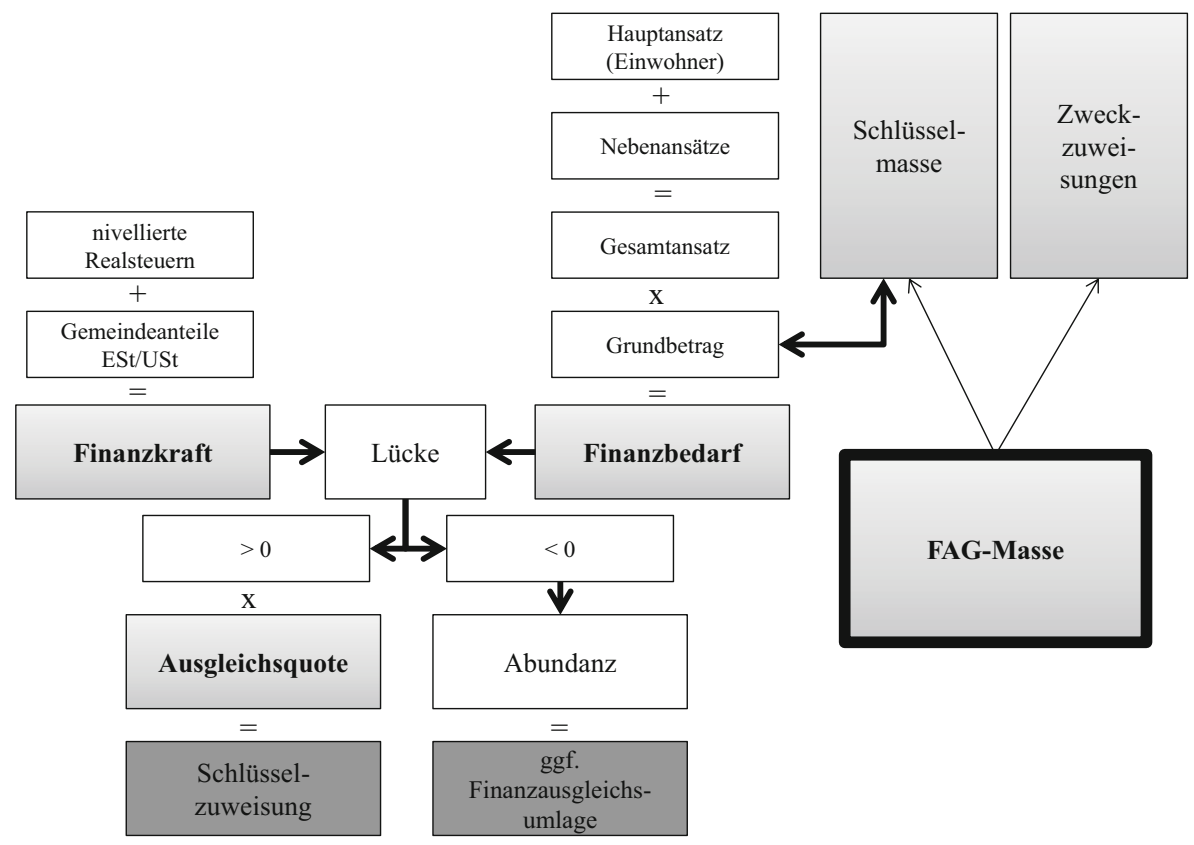

Abb. 7 Funktionsweise des Kommunalen Finanzausgleichs. Quelle: Lenk et al. 2019b, S. 344 
erhält diese Schlüsselzuweisungen. Die resultierende Lücke wird in Höhe der Ausgleichsquote geschlossen. Folglich werden mittels Finanzkraft, Finanzbedarf und Ausgleichsquote die relativen Gewichte der Kommunen an der (Teil-) Schlüsselmasse in einem Dreiecksverhältnis zueinander bestimmt (Lenk et al. 2019b, S. $344 \mathrm{ff}$.).

\subsection{Rolle des demografischen Wandels bei der Finanzbedarfsbestimmung}

Die Länder berücksichtigen die kommunalen Sonderbedarfe hinsichtlich des demografischen Wandels bisher in unterschiedlichem Maße. Generell ist hier zunächst darauf hinzuweisen, dass die meisten Flächenländer über eine sog. Hauptansatzstaffel verfügen, auf deren Basis mit ansteigender Einwohnerzahl i.d.R. höhere Veredelungsfaktoren und demzufolge pauschale Mehrbedarfe unterstellt werden. In der Folge wird keine proportionale Beziehung zwischen der Bevölkerungszahl und dem Finanzbedarf unterstellt, sondern einem mit der Einwohnerzahl in logarithmischer Form wachsenden Finanzbedarf ausgegangen. Bezogen auf den Bevölkerungsrückgang unterstellt dies, dass die Pro-Kopf-Ausgaben überproportional abnehmen müssten, was das Gegenteil von Kostenremanenzen widerspiegelt (Starke 2020, S. 225). Dabei bezieht sich die Hauptansatzstaffel im Kern vor allem auf die Unterschiede im Niveau der Einwohnerzahl und nicht auf ihre Dynamik im Zeitverlauf, weshalb größere Kommunen aufgrund ihrer im Vergleich zu kleineren Kommunen höheren Bedarfsmesszahl mehr Schlüsselzuweisungen erhalten, während kleinere Gemeinden benachteiligt werden (Bogumil et al. 2013, S. 260). Aus diesem Grund repräsentiert die Hauptansatzstaffel zugleich eine Teilursache für die finanziellen Probleme peripher gelegener Gemeinden.

Um die Effekte des demografischen Wandels im System der Schlüsselzuweisungen zu dämpfen, kommen Nebenansätze für die Altersstruktur oder die Bevölkerungsschrumpfung zur Anwendung. Erstere geben einen Aufschlag auf die Einwohnerzahl in Abhängigkeit der Anzahl von Kleinkindern oder Schülern. Gemeinden mit einer überdurchschnittlich hohen Quote an Klein- oder Schulkindern wird demnach eine höhere Bedarfsmesszahl zugesprochen, wodurch sie c.p. mehr Schlüsselzuweisungen erhalten. Ansätze zur Veredelung des Bevölkerungsrückgangs bzw. Demografieansätze stellen i.d.R. simple Modifikationen des Hauptansatzes dar: Dabei wird die tatsächliche Einwohnerzahl einer Gemeinde mit einer (mehrere Jahre umfassenden) durchschnittlichen Einwohnerzahl verglichen. Der größere der beiden Werte wird schließlich im Hauptansatz für die Berechnung des Finanzbedarfs herangezogen. Dadurch wird dem überproportionalen Rückgang des kommunalen Finanzbedarfs in schrumpfenden Gemeinden, der durch den Hauptansatz bzw. die Hauptansatzstaffel induziert wird, in Teilen entgegengewirkt. Demografieansätze sind in der finanzwissenschaftlichen Literatur generell umstritten (Starke 2020, S. 240 ff.): Lenk et al. (2012b, S. 107 f.) kritisieren etwa, dass die Kostenremanenzeffekte nicht direkt in die Finanzbedarfsermittlung eingehen würden, sondern nur über eine Modifikation der Einwohnerzahl abgebildet werden. Schrumpft die Einwohnerzahl einer Gemeinde stetig, dann wird der im Rahmen des Demografieansatzes generierte Puffer im Zeitverlauf recht schnell aufgezehrt. Eine stärker nivellierende Entwicklung resultiert stattdessen in Gemeinden mit einem sprunghaften Verlauf der Bevölkerungsentwicklung. Eine positive Wirkung bei stetig schrumpfenden Kom- 
munen tritt dagegen lediglich in den ersten Jahren der Einführung eines Demografiefaktors ein (ausführlicher Färber et al. 2012, S. 103). Auf dieser Kritik aufbauend wurden in Hessen und Mecklenburg-Vorpommern alternative Demografiefaktoren implementiert, die den Finanzbedarf der Gemeinden mit einem überdurchschnittlichen Bevölkerungsrückgang erhöhen und dabei auf den konkreten Rückgang ihrer Einwohnerzahl zurückgehen (Starke 2020, S. 245 ff.).

\subsection{Rückwirkungen der empirischen Ergebnisse}

Die empirischen Ergebnisse haben gezeigt, dass (milde bis erhebliche) Kostenremanenzen und die Veränderung der Altersstruktur die Gemeindehaushalte vor umfangreiche Belastungen stellen. Da sich die Kostenremanenzeffekte nicht nur in den bereinigten Ausgaben, sondern auch in den Zuschussbeträgen manifestieren, entfalten sie Rückwirkungen, die im Rahmen des Kommunalen Finanzausgleichs anerkannt werden sollten. Es stellt sich daher erstens die Frage, wie die statistisch nachgewiesenen Kostenremanenzen zu deuten sind und wie ein entsprechender Ergänzungsansatz im Schlüsselzuweisungssystem aussehen könnte. Zweitens ist relevant, ob eine Hauptansatzstaffel auf der einen und Nebenansätze für den Bevölkerungsrückgang und für die Altersstruktur auf der anderen Seite grundsätzlich koexistieren können.

Bezüglich der ersten Fragestellung stellt der bisher praktizierte Demografie-Nebenansatz eine sehr gute Basis dar: Laut $\S 17$ Abs. 5 FAG M-V wird die Entwicklung der Einwohnerzahl einer Gemeinde (ohne zentralörtlichen Status) über einen Zeitraum von 10 Jahren der durchschnittlichen Bevölkerungsentwicklung aller Gemeinden gegenübergestellt. Fällt die Schrumpfung der Einwohnerzahl in der Gemeinde größer aus als im Durchschnitt aller Gemeinden, dann wird der überdurchschnittliche Teil des Einwohnerverlusts mit dem Faktor 0,35 multipliziert und der Einwohnerzahl der schrumpfenden Gemeinde bei der Finanzbedarfsbestimmung zugerechnet, wodurch diese c.p. mehr Schlüsselzuweisungen erhält.

Auf Grundlage der Resultate würde sich die Stärke der Veredelung deutlich erhöhen (auf Basis des Fixed Effects-Modells mit Wachstumsrate etwa auf den Faktor $0,91)$ und sich zudem auf einen Zeitraum von 16 Jahren beziehen. Folglich würde ein größerer Teil der verlorenen Einwohner in schrumpfenden Gemeinden zur fiktiven Einwohnerzahl bei der Bestimmung des Finanzbedarfs hinzugerechnet. Daran anknüpfend könnten die Ergebnisse der geografisch gewichteten Regression weiterverwendet werden: Möglich wären hierbei regionsspezifische Veredelungsfaktoren, die auf Basis der Relation der lokalen Regressionskoeffizienten in Abb. 6 ermittelt werden könnten. Die unterschiedliche Intensität der Kostenremanenzen kann dadurch in Abhängigkeit ihrer geografischen Lage im Kommunalen Finanzausgleich Berücksichtigung finden. Folglich würden Gemeinden mit einem höheren Bevölkerungsrückgang aufgrund ihrer höheren Veredelungsfaktoren c.p. höhere Finanzbedarfe aufweisen. Schwieriger ist zu beurteilen, ob die geforderte Einfachheit des Kommunalen Finanzausgleichs durch die Dichte an gemeindespezifisch zu berücksichtigenden Informationen zu sehr vermindert wird. Alternativ zu differenziert nach der Stärke der Kostenremanenzen zugeschnittenen Regionen könnten auch landkreisspezifische Veredelungsfaktoren berechnet werden, auch wenn Landkreisgrenzen nur bedingt ein Indikator für die Stärke der Remanenzeffekte zu sein scheinen. 
Mit Blick auf die zweite und letzte Fragestellung besteht durch die Logik einer Hauptansatzstaffel ein gewisser Widerspruch zum Nebenansatz für den Bevölkerungsrückgang, da sie pauschale Mehrbedarfe mit ansteigender Einwohnerzahl unterstellt, was einem mit der Einwohnerzahl in logarithmischer Form wachsenden Pro-Kopf-Finanzbedarf entspricht. Dieser Widerspruch ist jedoch mit Blick auf die Bezugsgrößen sehr gut lösbar: Die Hauptansatzstaffel unterscheidet die Statusgröße, nach der eine große Gemeinde c.p. eine größere Bedarfsmesszahl verzeichnet als eine kleine Gemeinde. Dieser Tatbestand gilt auch dann, wenn die Einwohnerzahlen beider Gemeinden sinken. Für den Umfang der Kostenremanenzeffekte ist allerdings der Umfang der Schrumpfung relevant, der sich nicht auf die Statusgröße der Einwohnerzahl, sondern die Entwicklung der Einwohnerzahl im Zeitverlauf bezieht. Somit kann ein Ergänzungsansatz für den Rückgang der Einwohnerzahl neben einer Hauptansatzstaffel existieren, da sie verschiedene Bedarfstatbestände bedienen und sich demnach nicht gegenseitig ausschließen.

Ein Zusammenwirken der Veredelung des Bevölkerungsrückgangs und der Altersstruktur ist ebenfalls möglich und wird durch die verschiedenen Modelle angezeigt. Die auf den Ist-Stand der Variablen bezogenen Regressionskoeffizienten geben die durch die Altersstrukturkostenprofile illustrierten Mehrbelastungen durch jüngere Altersklassen und Minderbelastungen durch ältere Alterskohorten wieder. Dabei besteht die Möglichkeit, getrennte Nebenansätze für Kleinkinder und Schüler zu schaffen, um die hohen Lasten durch die Kinderbetreuung und dagegen nur moderaten Belastungen durch die Schulträgeraufgaben sachgerecht anzuerkennen.

Zudem ist die Frage, wie die Resultate der Regressionsmodelle mit Wachstumsraten in den Kommunalen Finanzausgleich implementiert werden können. So werden die Effekte des Bevölkerungsrückgangs durch diese Modelle recht eindeutig wiedergegeben; bezugnehmend auf die Altersstruktur (mit Ausnahme des Anteils der 6- bis 18-Jährigen) erzielen sie aber so gut wie keinen statistischen Mehrwert. Sie repräsentieren deshalb ein Indiz dafür, dass die demografischen Belastungen sowohl auf das Niveau der Pro-Kopf-Zuschussbeträge und -Ausgaben als auch deren jährliche Entwicklung einwirken. Des Weiteren bestätigen sie die im Fixed Effects-Modell mit Ist-Ständen erzielten Ergebnisse und dienen darauf aufbauend als Richtlinien, wie stark die Mehrbelastungen der Gemeinden durch den Bevölkerungsrückgang bei der Bestimmung des Finanzbedarfs im System der Schlüsselzuweisungen berücksichtigt werden sollen.

\section{Fazit und Ausblick}

Der demografische Wandel stellt die Kommunen einerseits aufgrund von Altersstruktureffekten (ausgaben- und zuschussbetragssteigernde Effekte durch Jugendlastigkeit, eher entlastende Effekte durch eine ansteigende Quote an älteren Menschen) und andererseits wegen Kostenremanenzeffekten vor vielgestaltige Herausforderungen. Letztere entfalten sich in den Bereichen der technischen und sozialen Infrastruktur, wobei üblicherweise von milden oder erheblichen Kostenremanenzen ausgegangen wird. Im ersten Fall sinken die Gesamtausgaben infolge des Bevölkerungsrückgangs zwar unterproportional, jedoch steigen die Pro-Kopf-Ausgaben. Bei 
erheblichen Kostenremanenzen steigen die Gesamtausgaben bei rückläufiger Bevölkerung sogar an, weshalb die Pro-Kopf-Ausgaben noch weitaus stärker zunehmen. Erforderliche Anpassungsmaßnahmen, wie der Rückbau bestimmter kommunaler Infrastrukturen, werden dabei zum einen durch die fehlende Akzeptanz der Bürger vor Ort und zum anderen durch das schleichende Auftreten der Effekte erschwert.

Eine Möglichkeit, die durch den demografischen Wandel hervorgerufenen Lasten in den Gemeinden und Städten zu mindern, ist durch den Kommunalen Finanzausgleich gegeben. Dieser hat die Aufgabe, den Kommunen eine aufgabenangemessene Finanzausstattung zur Verfügung zu stellen.

Die empirischen Untersuchungen hinsichtlich der Gemeinden und Städte Mecklenburg-Vorpommerns zeigen, dass dem Bedarfstatbestand des demografischen Wandels künftig eine deutlich größere Rolle im Kommunalen Finanzausgleich zugestanden werden sollte als dies bislang der Fall ist. So resultieren der stetige Rückgang der Einwohnerzahlen und die Veränderung der Altersstruktur in den Kommunen Mecklenburg-Vorpommerns in einem höheren Niveau und Wachstum von Pro-KopfZuschussbeträgen und -Ausgaben. Die in unterschiedlichen Modelltypen nachgewiesenen Kostenremanenzeffekte fallen den Ergebnissen der geografisch gewichteten Regression zufolge in Gemeinden mit einem höheren Bevölkerungsrückgang nochmals stärker aus. Mit Blick auf den Kommunalen Finanzausgleich sollten die demografischen Sonderbedarfe der Gemeinden gezielt bei der Bestimmung des Finanzbedarfs im Schlüsselzuweisungssystem veredelt werden. Dabei bietet es sich an, den Bevölkerungsrückgang nach geografischer Lage und folglich nach Stärke der jeweiligen Kostenremanenzeffekte unterschiedlich stark zu veredeln.

Für künftige (empirische) Untersuchungen verbleibt die Frage, ob neben den technischen Kostenremanenzeffekten auch die politischen Remanenzeffekte im Finanzausgleich berücksichtigt werden sollten. Eine Unterscheidung nach diesen beiden Arten von Remanenzeffekten war im Rahmen der empirischen Untersuchung nicht möglich. Somit wäre denkbar, die kommunalen Aufgaben entsprechend des Produktrahmenplans stärker zu differenzieren und somit eine grobe Zuteilung zu technischen und politischen Kostenremanenzeffekten zu ermöglichen.

Funding Open Access funding enabled and organized by Projekt DEAL.

Open Access Dieser Artikel wird unter der Creative Commons Namensnennung 4.0 International Lizenz veröffentlicht, welche die Nutzung, Vervielfältigung, Bearbeitung, Verbreitung und Wiedergabe in jeglichem Medium und Format erlaubt, sofern Sie den/die ursprünglichen Autor(en) und die Quelle ordnungsgemäß nennen, einen Link zur Creative Commons Lizenz beifügen und angeben, ob Änderungen vorgenommen wurden.

Die in diesem Artikel enthaltenen Bilder und sonstiges Drittmaterial unterliegen ebenfalls der genannten Creative Commons Lizenz, sofern sich aus der Abbildungslegende nichts anderes ergibt. Sofern das betreffende Material nicht unter der genannten Creative Commons Lizenz steht und die betreffende Handlung nicht nach gesetzlichen Vorschriften erlaubt ist, ist für die oben aufgeführten Weiterverwendungen des Materials die Einwilligung des jeweiligen Rechteinhabers einzuholen.

Weitere Details zur Lizenz entnehmen Sie bitte der Lizenzinformation auf http://creativecommons.org/ licenses/by/4.0/deed.de. 


\section{Literatur}

Auer, Benjamin, und Horst Rottmann. 2015. Statistik und Ökonometrie für Wirtschaftswissenschaftler. Wiesbaden: Springer.

Bauer, David, und Xenia Frei. 2017. Der Demographieansatz im kommunalen Finanzausgleich. ifo Dresden berichtet 24:20-24.

Bogumil, Jörg, Sascha Gerber, und Maren Schickentanz. 2013. Handlungsmöglichkeiten kommunaler Demografiepolitik. In Demografiepolitik, Hrsg. Michael Hüther, 259-282. Wiesbaden: Springer VS.

Borge, Lars-Erik, und Jørn Rattsø. 1995. Demographic shift, relative costs and the allocation of local public consumption in Norway. Regional Science and Urban Economics 25:705-726.

Brunsdon, Chris, Alexander Stewart Fotheringham, und Martin Charlton. 1998. Geographically weighted regression - modelling spatial non-stationarity. Journal of the Royal Statistical Society (Series D The Statistician) 47:431-443.

Bundesministerium für Verkehr, Bau und Stadtentwicklung (BMVBS). 2007. Die demografische Entwicklung in Ostdeutschland und ihre Auswirkungen auf die öffentlichen Finanzen. Forschungen 128. https://www.bbsr.bund.de/BBSR/DE/veroeffentlichungen/ministerien/bmvbs/forschungen/ 2007/Heft128_DL.pdf. Zugegriffen: 22. März 2021.

Büttner, Thiess, Fédéric Holm-Hadulla, Rüdiger Parsche, und Christiane Starbatty. 2008. Analyse und Weiterentwicklung des Kommunalen Finanzausgleichs in Nordrhein-Westfalen. Ifo Forschungsberichte 41. https://www.ifo.de/DocDL/ifo_Forschungsberichte_41.pdf. Zugegriffen: 18. März 2021.

Dobroschke, Stephan, Jens-Martin Gutsche, und Michael Thöne. 2014. Ermittlung von aufgabenbezogenen Kostenremanenzen im Rahmen des kommunalen Finanzausgleichs in Sachsen-Anhalt. FifoBerichte 15. http://www.fifo-koeln.org/images/stories/fifo-bericht\%20nr\%20\%2015_kmpr.pdf. Zugegriffen: 18. März 2021.

Eck, Alexander, Joachim Ragnitz, Johannes Steinbrecher, und Christian Thater. 2012. Evaluation der Zukunftsfestigkeit des Gleichmässigkeitsgrundsatzes II im kommunalen Finanzausgleich des Freistaates Sachsen. ifo Dresden Studien 67. https://www.ifo.de/DocDL/ifo_Dresden_Studien_67.pdf. Zugegriffen: 17. März 2021.

Eckey, Hans-Friedrich, Reinhold Kosfeld, und Matthias Türck. 2007. Regional convergence in Germany: a geographically weighted regression approach. Spatial Economic Analysis 2:45-64.

Ewringmann, Dieter. 1971. Zur Voraussage kommunaler Investitionsbedarfe - Ein Beitrag zur positiven Theorie öffentlicher Bedarfe. Wiesbaden: VS.

Färber, Gisela, Joachim Wieland, Johanna Wolff, Marco Salm, und Dirk Zeitz. 2012. Reform des kommunalen Finanzausgleichs in Thüringen. Speyerer Forschungsberichte 271. https://d-nb.info/ 1031536876/34. Zugegriffen: 17. März 2021.

Fernandez, Raquel, und Richard Rogerson. 2001. The determinants of public education expenditures: longer-run evidence from the states. Journal of Education Finance 27:567-583.

Fotheringham, Alexander Stewart, Chris Brunsdon, und Martin Charlton. 2010. Geographically weighted regression-the analysis of spatially varying relationships. Chichester: Wiley.

Frei, Xenia, Joachim Ragnitz, und Felix Rösel. 2018. Kosten kommunaler Leistungserstellung unter dem Einfluss von demografischem Wandel und Urbanisierung. ifo Dresden Studien 80. https://www.ifo. de/publikationen/2018/monographie-autorenschaft/kosten-kommunaler-leistungserstellung-unterdem. Zugegriffen: 19. März 2021.

Freigang, Dirk. 2012. Auswirkungen der demografischen Entwicklungen auf den kommunalen Sektor in Ost- und Westdeutschland. Technische Universität Dresden: Dissertation.

Fábián, Zsófia. 2014. Method of the geographically weighted regression and an example for its application. Regional Statistics 4:61-75.

Gnädinger, Marc. 2012. Generationengerechte Haushalts- und Finanzpolitik in Kommunen - Haushaltsausgleich, -konsolidierung und Schuldenabbau bis zur Schuldenfreiheit in Städten und Gemeinden. Taunusstein: Driesen.

von Gottl-Ottlilienfeld, Friedrich. 1928. Bedarf und Deckung - Ein Vorgriff in Theorie der Wirtschaft als Leben. Jena: Gustav Fischer.

Grob, Ueli, und Stefan Wolter. 2005. Demographic change and public education spending: a conflict between young and old? Education Economics 15:277-292.

Grüttner, André, und Mario Hesse. 2013. Einfluss des demografischen Wandels auf die Kommunalfinanzen und das Instrument des Demografieansatzes im Kommunalen Finanzausgleich - Evidenz aus einer Studie für das Land Hessen. In Der demografische Wandel - Eine Gefahr für die Sicherung gleichwertiger Lebensbedingungen?, Hrsg. Bundesinstitut für Bau-, Stadt- und Raumforschung (BBSR) 
im Bundesamt für Bauwesen und Raumordnung (BBR), 52-63. Bonn: Bundesamt für Bauwesen und Raumordnung.

Gutsche, Jens-Martin. 2006. Soziale Infrastrukturen: Anpassungsfähigkeit und Remanenzkosten bei Nachfrageveränderungen. Informationen zur Raumentwicklung 5:271-280.

von Hauff, Michael, und Bülent Tarkan. 2006. Die finanzwirtschaftliche Situation der kommunalen Ebene - Die demografische Perspektive. Volkswirtschaftliche Diskussionsbeiträge an der Technischen Universität Kaiserslautern, Bd. 23

Hesse, Mario, und Tim Starke. 2018. Kommunaler Finanzbedarf - Können kommunale Bedarfe überhaupt präzise bestimmt werden? Jahrbuch für öffentliche Finanzen 241(2):181-196.

Junkernheinrich, Martin. 1992. Sonderbedarfe im kommunalen Finanzausgleich. Berlin: Analytica.

Kelley, Allen. 1976. Demographic change and the size of the government sector. Southern Economic Journal 43:1056-1066.

Kops, Manfred. 1989. Möglichkeiten und Restriktionen einer Berücksichtigung von Sonderbedarfen im Länderfinanzausgleich. Wiesbaden: VS.

Ladd, Helen, und Sheila Murray. 2001. Intergenerational conflict reconsidered: county demographic structure and the demand for public education. Economics of Education Review 20:343-357.

Lenk, Thomas, und Tim Starke. 2015. Auswirkungen der demografischen Entwicklung auf die öffentlichen Finanzen, Arbeitspapiere des Instituts für öffentliche Finanzen und Public Management 48. https://www.wifa.uni-leipzig.de/fileadmin/Fakultät_Wifa/Institut_für_öffentliche_Finanzen_und_ Public_Management/48_Lenk-Starke_-_Auswirkungen_der_demografischen_Entwicklung_auf_ die_oeffentlichen_Finanzen.pdf. Zugegriffen: 17. März 2021.

Lenk, Thomas, Mario Hesse, André Grüttner, und Tim Reichardt. 2012a. Finanzwissenschaftliches Gutachten zur Fortschreibung des Kommunalen Finanzausgleichs in Hessen. https://www.wifa.unileipzig.de/fileadmin/Fakultät_Wifa/Institut_für_öffentliche_Finanzen_und_Public_Management/ 121213_Gutachten_KFA_Hessen_Gesamtdokument_web.pdf. Zugegriffen: 18. März 2021.

Lenk, Thomas, Mario Hesse, und Florian Woitek. 2012b. Finanzwissenschaftliches Gutachten zur Fortschreibung des Kommunalen Finanzausgleichs in Brandenburg. https://www.wifa.uni-leipzig. de/fileadmin/Fakultät_Wifa/Institut_für_öffentliche_Finanzen_und_Public_Management/Lenk Hesse_et_al_2012_-_Finanzwissenschaftliches_Gutachten_zur_Fortschreibung_2_.pdf. Zugegriffen: 18. März 2021.

Lenk, Thomas, Mario Hesse, Tim Starke, Florian Woitek, und André Grüttner. 2017. Überprüfung des kommunalen Finanzausgleichs in Mecklenburg-Vorpommern. https://www.wifa.uni-leipzig.de/ fileadmin/Fakultät_Wifa/Institut_für_öffentliche_Finanzen_und_Public_Management/Lenk_Hesse_ Starke_et_al_2017_Gutachten_KFA_M-V.pdf. Zugegriffen: 17. März 2021.

Lenk, Thomas, Mario Hesse, und Tim Starke. Finanzwissenschaftliche Analysen und finanzwirtschaftliche Berechnungen im Rahmen des Kommunalen Finanzausgleichs in Mecklenburg-Vorpommern. 2018. https://www.wifa.uni-leipzig.de/fileadmin/Fakult\%C3\%A4t_Wifa/Institut_f\%C3\%BCr_\%C3 \%B6ffentliche_Finanzen_und_Public_Management/Lenk_Hesse_Starke_2018_Finanzwissenschaft liche_Analyse_zum_FAG_M-V_web.pdf. Zugegriffen: 18. März 2021.

Lenk, Thomas, Mario Hesse, und Tim Starke. 2019a. Gemeindefinanzbericht Sachsen 2018/2019. Sachsenlandkurier, Bd. 6

Lenk, Thomas, Tim Starke, und Mario Hesse. 2019b. Kommunaler Finanzausgleich - vertikale und horizontale Verteilung, Strukturprinzipien und Wirkungen. In Haushalts- und Finanzwirtschaft der Kommunen in der Bundesrepublik, Hrsg. Tilmann Schweisfurth, Walter Wallmann, 325-358. Berlin: Berliner Wissenschafts-Verlag.

Lenk, Thomas, Tim Starke, und Mario Hesse. 2021. Überprüfung des Kommunalen Finanzausgleichs in Brandenburg zum Ausgleichsjahr 2022. https://mdfe.brandenburg.de/sixcms/media.php/9/Lenk $\% 20$ Hesse $\% 20$ Starke $\% 202021 \% 20$ Gutachten $\% 20$ zur\%20\%C3\%9Cberpr\%C3\%BCfung\%20des \%20KFA\%20in\%20Brandenburg.pdf. Zugegriffen: 25. Juni 2021.

Poterba, James. 1996. Demographic structure and the political economy of public education. Cambridge: National Bureau of Economic Research.

Sachverständigenrat zur Begutachtung der Gesamtwirtschaftlichen Entwicklung. 2004. Erfolge im Ausland - Herausforderungen im Inland - Jahresgutachten 2004/05. Wiesbaden: Sachverständigenrat zur Begutachtung der Gesamtwirtschaftlichen Entwicklung.

Seitz, Helmut. 2007. The impact of demographic change on fiscal policy in Germany. In Demographic change in Germany, Hrsg. Ingrid Hamm, Helmut Seitz, und Martin Werding, 129-164. Berlin, Heidelberg: Springer. 
Seitz, Helmut. 2008. Die Demographieabhängigkeit der Ausgaben und Einnahmen der öffentlichen Haushalte - Eine empirische Analyse unter Berücksichtigung der föderalen Verflechtungen. Gütersloh: Bertelsmann Stiftung.

Seitz, Helmut, Thomas Fester, Andreas Kappeler, und Marcel Thum. 2004. Öffentliche Infrastruktur und kommunale Finanzen. Bonn: Bundesamt für Bauwesen und Raumordnung.

Seitz, Helmut. 2004. Implikationen der demographischen Veränderungen für die öffentlichen Haushalte und Verwaltungen. https://www.econstor.eu/bitstream/10419/22712/1/ddpe200408.pdf. Zugegriffen: 18. März 2021. Dresden Discussion Paper in Economics 8.

Siedentop, Stefan, Moritz Hans, und Martin Schulwitz. 2015. Kommunale Infrastrukturkosten und Demographie. Dortmund, Gütersloh: Bertelsmann Stiftung.

Siedentop, Stefan, Georg Schiller, Jens-Martin Gutsche, Matthias Koziol, und Jörg Walther. 2006. Infrastrukturkostenrechnung in der Regionalplanung - Ein Leitfaden zur Abschätzung der Folgekosten alternativer Bevölkerungs- und Siedlungsszenarien für soziale und technische Infrastrukturen. Berlin, Bonn: Bundesamt für Bauwesen und Raumordnung.

Starke, Tim. 2020. Bestimmung des Finanzbedarfs von Gemeinden und Städten im horizontalen Koтmunalen Finanzausgleich mit Fokus auf die demografische Entwicklung. Berlin: Berliner WissenschaftsVerlag.

Wissenschaftlicher Beirat beim Bundesministerium der Finanzen. 2013. Finanzpolitische Herausforderungen des demografischen Wandels im föderativen System. Berlin: Bundesministerium für Finanzen.

Zimmermann, Horst, Klaus-Dirk Henke, und Michael Broer. 2017. Finanzwissenschaft - Eine Einführung in die Staatsfinanzen. München: Vahlen.

Zokalj, Mateo. 2016. The impact of population aging on public finance in the European Union. Financial Theory and Practice 40:383-412. 